\title{
Script Geometry
}

\author{
P. Cerejeiras, U. Kähler, F. Sommen and A. Vajiac
}

\begin{abstract}
In this paper we describe the foundation of a new kind of discrete geometry and calculus called Script Geometry. It allows to work with more general meshes than classic simplicial complexes. We provide the basic definitions as well as several examples, like the Klein bottle and the projective plane. Furthermore, we also introduce the corresponding Dirac and Laplace operators which should lay the groundwork for the development of the corresponding discrete function theory.
\end{abstract}

Mathematics Subject Classification (2010). 30G35, 65N30, 58A14.

Keywords. script, simplicial topology, discrete exterior derivative, HodgeDirac operator, discrete Laplacian, cohomology.

\section{Introduction}

In the last two decades one can observe an ever increasing interest in the analysis of discrete structures. On one hand the fact that nowadays everybody can harness large computational power, but the computer is restricted to work with discrete values only, created an increased interest in working with discrete structures. This is true even for persons who are originally unrelated to the field. An outstanding example can be seen in the change of the philosophy of the Finite Element Method.

From the classical point of view the finite element method is essentially a method for discretization of partial differential equations via a variational formulation, i.e. one first establishes the variational formulation and discretizes the problem by creating ansatz spaces via introducing a mesh (normally by triangularization) and (spline) functions defined over the mesh. One of the major problems with this approach is that there is no a priori connection between the choice of the mesh and the variational formulation. The modern approach lifts the problem and, therefore, the finite element modelation directly on to the mesh, resulting in the so-called Finite Element Exterior Calculus $[1,14]$. Hereby, one chooses first the mesh and introduces a boundary operator given by the mesh which induces the corresponding discrete 
variational formulation. From a practical point of view this is even more interesting since finite element meshes are also widely being applied in other fields, such as computer graphics [13], [14]. In this framework notions of discrete vector fields and operators acting on them, e.g. discrete divergence and curl, appear in a rather canonical way instead of being introduced artificially by additional discretizing a continuous formulation. This also leads to immediate applications such as the problem of discrete Hodge decompositions of $3 \mathrm{D}$ vector fields on irregular grids. In this context one can also study the notion of a Dirac operator [27].

Yet, if we look at the literature the existing theory is based on working with simplicial complexes and triangularizations [13]. But in FEM or in computer graphics more irregular meshes (in the sense that they do not work with simplicial complexes) are widely used. Furthermore, there are problems in other fields like problems in physics which are traditionally modeled by means of a continuous analysis which are more and more directly studied on the discrete level, the principal example being the Ising model from statistical physics as opposed to the continuous Heisenberg model. These models require a discrete function theory to work with them, similar to the $2 \mathrm{D}$-case where discrete complex analysis plays a major role. In fact most of the recent advances on the 2D-Ising model by S. Smirnov and his collaborators are based on a clever interaction between classic and discrete complex analysis [29]. This is possible since discrete complex analysis is under (more or less) constant development since the forties [25, 28].

Unfortunately, the same cannot be said about the higher-dimensional case. While lately one can observe several approaches to create a discrete function theory in higher dimensions based on lattice discretizations of the Dirac operator (see $[32,26,21,18,19,4,8]$ ) they are closer in spirit to finite difference methods than finite element methods $([5,22,23,2,6])$. Nevertheless, these approaches lead to a well established function theory $[17,9,10$, $11,12,20,7]$. For a function theory in connection with the above mentioned finite element exterior calculus we do not want to be restricted to meshes coming from simplicial complexes. Therefore, one needs a new kind of geometry which allows to work directly with general meshes.

In this paper we are going to lay the foundations of a new type of discrete geometry called script geometry which is not restricted to simplicial complexes. After a short review of simplicial topology we define the principal objects as well as introducing the corresponding Dirac and Laplace operators as discrete versions of the abstract Hodge-Dirac operator. Furthermore, to give a more clear understanding of what we are aiming at we are going to present several examples, such as the Möbius strip, the Klein bottle, the torus, and the projective plane. It is our modest hope that the presented framework will be interesting enough to be explored by many mathematicians in the future. 


\section{Brief review of simplicial topology}

An abstract simplicial complex is a collection $\mathcal{S}$ of finite non-empty sets, such that if $A$ is an element of $\mathcal{S}$, then every non-empty subset of $A$ is also an element of $\mathcal{S}$. An element $A$ of $\mathcal{S}$ is called a simplex of $\mathcal{S}$; its dimension is one less than the number of its elements, and each non-empty subset of $A$ is called a face of $A$. Vertices of $\mathcal{S}$ are the one-point elements $v$ of $\mathcal{S}$, and $\{v\}$ is by definition a 0 -simplex.

If $K$ is a topological simplicial complex and $V$ its vertex set, then the collection of all subsets $\left\{a_{0}, \ldots, a_{n}\right\}$ of $V$ such that the vertices $a_{0}, \ldots, a_{n}$ span a simplex of $K$, is called the vertex scheme of $K$. The vertex scheme of a topological simplicial complex is an example of abstract simplicial complex. In fact, every abstract complex $\mathcal{S}$ is isomorphic to the vertex scheme for some simplicial complex $K$, called also the geometric realization of $\mathcal{S}$, uniquely determined up to a linear isomorphism.

Let $\sigma$ be an abstract simplex. Two orderings of its vertex set are equivalent if they differ by an even permutation. There are two equivalence classes (in dimensions bigger than 1), each one of them called an orientation of $\sigma$. For 0-simplexes, there is only one orientation.

If $K$ is a simplicial complex, then a $p$-chain on $K$ is a function $c$ from the set of oriented $p$-simplices of $K$ to $\mathbb{Z}$, such that: (a) $c(\sigma)=-c(-\sigma)$; and (b) $c(\sigma)=0$ for all but finitely many oriented $p$-simplices $\sigma$. Addition of oriented $p$-chains is done by adding their integer values. The resulting group is denoted by $C_{p}(K)$.

If $\sigma$ is an oriented simplex, the elementary chain $c$ corresponding to $\sigma$ is the function defined as follows: (a) $c(\sigma)=1$, (b) $c(-\sigma)=-1$, and (c) $c(\tau)=0$ for all other oriented simplices. The usual convention denotes by $\sigma$ both the oriented simplex and its elementary $p$-chain $c$. This allows the notation $-\sigma$ for the simplex with opposite orientation than $\sigma$.

A well-known result is that $C_{p}(K)$ is a free Abelian group, a basis is obtained by orienting each $p$-simplex and using the corresponding elementary chains as a basis. Therefore, with the exception of $C_{0}(K)$, the groups $C_{p}(K)$ have no natural basis, as one must orient the $p$-simplices in $K$ in an arbitrary fashion to obtain a basis.

The homomorphism of groups:

$$
\partial_{p}: C_{p}(K) \rightarrow C_{p-1}(K)
$$

is called the boundary operator, defined by

$$
\partial_{p}\left[v_{0}, \ldots, v_{p}\right]:=\sum_{i=0}^{p}(-1)^{i}\left[v_{0}, \ldots, \widehat{v}_{i}, \ldots, v_{p}\right]
$$

where the hat means deletion from the array. The operator $\partial_{p}$ is well-defined and it has the property

$$
\partial_{p}(-\sigma)=-\partial_{p}(\sigma)
$$


for all simplices $\sigma$. For example:

$$
\partial_{1}\left[v_{0}, v_{1}\right]=v_{1}-v_{0}, \quad \partial_{2}\left[v_{0}, v_{1}, v_{2}\right]=\left[v_{1}, v_{2}\right]-\left[v_{0}, v_{2}\right]+\left[v_{0}, v_{1}\right]
$$

It can be proved that

$$
\partial_{p-1} \circ \partial_{p}=0
$$

so the kernel of $\partial_{p}$, denoted by $Z_{p}(K)$ is the group of $p$-cycles, and the image of $\partial_{p+1}$, denoted by $B_{p}(K)$, is the group of $p$-boundaries. The $p^{t h}$ homology group of $K$ is then defined as

$$
H_{p}(K):=Z_{p}(K) / B_{p}(K) .
$$

Cohomology is usually defined using the Hom functor. That makes cocycles to be "picket fences" inside triangularizations of manifolds.

\section{Script Geometry}

Let us start with the definition of our most basic object, the notion of a script.

Definition 3.1. A script is a collection

$$
\mathfrak{S}:=\left\{\mathfrak{S}_{-1}, \mathfrak{S}_{0}, \mathfrak{S}_{1}, \ldots, \mathfrak{S}_{k}, \ldots, \mathfrak{S}_{m}\right\}
$$

of sets $\mathfrak{S}_{k}$, the elements of which are called $k$-cells. In particular,

$$
\begin{aligned}
\mathfrak{S}_{-1} & :=\{\infty\}, \quad \mathfrak{S}_{0}:=\left\{p_{1}, \ldots, p_{j}, \ldots, p_{n_{0}}\right\}, \\
\mathfrak{S}_{1} & :=\left\{l_{1}, \ldots, l_{j}, \ldots, l_{n_{1}}\right\}, \quad \mathfrak{S}_{2}:=\left\{v_{1}, \ldots, v_{j}, \ldots, v_{n_{2}}\right\}, \ldots, \\
\mathfrak{S}_{k} & :=\left\{c_{1}^{k}, \ldots, c_{j}^{k}, \ldots, c_{n_{k}}^{k}\right\} .
\end{aligned}
$$

Traditionally 0,1 and $2-$ cells are called points, lines and planes, respectively.

Definition 3.2. A linear combination over $\mathbb{Z}$ of $k$-cells is called a $k$-chain:

$$
C_{k}:=\sum_{j} \lambda_{j} c_{j}^{k}, \quad \lambda_{j} \in \mathbb{Z},
$$

and we denote the module of $k$-chains by $\mathfrak{C}_{k}$. The boundary map $\partial$ from $\mathfrak{S}_{k}$ into $\mathfrak{C}_{k-1}$, the module of $(k-1)$-chains, is defined by:

$$
\partial c_{j}^{k}:=\sum_{s} \lambda_{j}^{k, s} c_{s}^{k-1},
$$

which naturally extends to the module $\mathfrak{C}_{k}$, and it is subject to $\partial^{2}=0$.

The support of a $k$-chain $C_{k}$ is the set of $k$-cells $c_{j}^{k}$ that are involved in the linear combination (3.2), i.e. for which $\lambda_{j}^{k} \neq 0$.

Let us remark that the coefficients $\lambda_{j}^{k, s}$ in (3.2) are uniquely determining the boundary operator $\partial$. For example, if $p^{0}$ is a 0 -chain, then if $p^{0}=\sum_{j} \lambda_{j} p_{j}$, we have:

$$
\partial p_{j}=1 \cdot \infty, \quad \partial p^{0}=\left(\sum_{j} \lambda_{j}\right) \cdot \infty
$$


Then $\partial p^{0}=0$ if and only if $\sum_{j} \lambda_{j}=0$. Since $\partial^{2}=0$ by definition, for a generic $k$-cell we have:

$$
0=\partial^{2} c_{j}^{k}=\partial\left(\sum_{s} \lambda_{j}^{k, s} c_{s}^{k-1}\right)=\sum_{\ell}\left(\sum_{s} \lambda_{j}^{k, s} \lambda_{s}^{k-1, \ell}\right) c_{\ell}^{k-2}
$$

therefore:

$$
\sum_{s} \lambda_{j}^{k, s} \lambda_{s}^{k-1, \ell}=0
$$

for all $\ell$.

Definition 3.3. A $k$-chain $C_{k}$ for which $\partial C_{k}=0$, it is generally called a $k$-cycle. A $k$-chain

$$
C_{k}=\sum_{j} \lambda_{j}^{k} c_{j}^{k}
$$

for which $\lambda_{j}^{k}= \pm 1$ is called an oriented surface, or simply a surface. A surface $C_{k}$ for which $\partial C_{k}=0$ is a closed surface.

Definition 3.4. A script $\mathfrak{S}$ for which every cell boundary $\partial c_{j}^{k}$ is a closed surface is called a geoscript.

Definition 3.5. A closed surface $C_{k}$ is called tight if and only if for every closed surface $C_{k}^{\prime}$ with $\operatorname{supp} C_{k}^{\prime} \subset \operatorname{supp} C_{k}$, it follows that $C_{k}^{\prime}= \pm C_{k}$, i.e. $C_{k}$ is the only closed surface, up to sign, with support inside $\operatorname{supp} C_{k}$.

A tight cell $c$ is a cell for which $\partial c$ is a closed tight surface. A geoscript is called tight if all its cells have a boundary which is a tight surface, i.e. all its cells are tight cells.

Any point $p_{j}$ is obviously tight. A line $l$ is tight if and only if $\partial l=p_{j}-p_{k}$, i.e. every tight line connects two points. Every plane $v$ which is tight has a boundary

$$
\partial v=\sum_{j=1}^{t} \lambda_{j} l_{j}, \quad \lambda_{j}= \pm 1,
$$

which forms a polygon, i.e. $\lambda_{j} \partial l_{j}=p_{j}-p_{j+1}$ whereby $p_{t+1}=p_{1}$, and all points $p_{1}, \ldots, p_{t}$ are different.

In Figure 1, we have drawn two examples of tight scripts and the far right one is a non-tight script. Please note that the "loop" script in Figure 1 is defined by:

$$
\begin{aligned}
& \mathfrak{S}_{0}=\left\{p_{0}, p_{1}\right\}, \quad \mathfrak{S}_{1}=\left\{l_{1}, l_{2}\right\}, \quad \mathfrak{S}_{2}=\{v\}, \\
& \partial l_{1}=p_{1}-p_{0}, \quad \partial l_{2}=p_{0}-p_{1}, \quad \partial v=l_{1}+l_{2} .
\end{aligned}
$$

Note that a tight geoscript of dimension $\leq 2$ is always topologically equivalent to a CW-complex. For higher dimensional geoscripts the situation can be more general than $\mathrm{CW}$-complexes.

The cells in a geoscripts are oriented cells and can each come in two states of orientation that are determined by the boundary map $\partial\left(c_{j}^{k}\right)$, i.e. if 

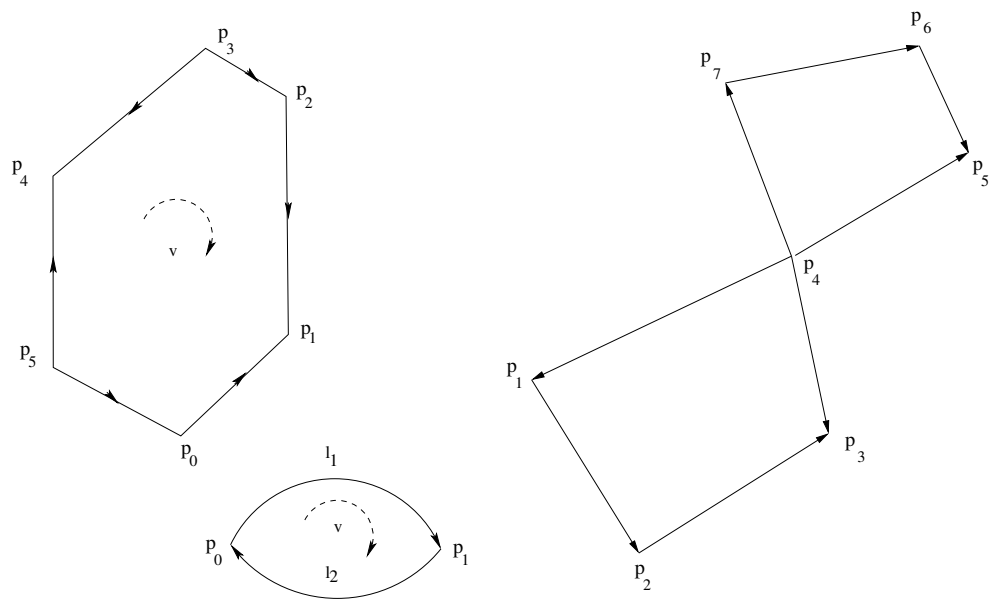

Figure 1. Examples of scripts

one replaces $c_{j}^{k}$ by $d_{j}^{k}=-c_{j}^{k}$ then also $\partial\left(d_{j}^{k}\right)=-\partial\left(c_{j}^{k}\right)$. But in general there could be more than two orientations on (closed surfaces inside) $\operatorname{supp} \partial\left(c_{j}^{k}\right)$ and so the mere knowledge of $\operatorname{supp} \partial\left(c_{j}^{k}\right)$ does not determine the orientations $\pm \partial\left(c_{j}^{k}\right)$. The tightness condition however ensures that on $\operatorname{supp} \partial\left(c_{j}^{k}\right)$ there can only be two states of closed orientation given by $\pm \partial\left(c_{j}^{k}\right)$, so that the state of orientation on each cell $c_{j}^{k}$ can be fully identified with the state of orientation on the boundary. The tightness condition also implies a number of interesting geometric properties for scripts, such as a line has two endpoints or a 2-cell is a polygon. In a forthcoming paper we prove that using tightness one can determine when a two-dimensional script corresponds to an oriented two-dimensional manifold.

Definition 3.6. A $k$-cell $c$ is called a $k$-simplex if either $c$ is a point (the case $k=0$ ), or the boundary $\partial c$ of $c$ is a tight $(k-1)$-surface that is the sum (with coefficients \pm 1$)$ of $k+1$ different $(k-1)$-cells that are also $(k-1)$-simplexes. A simplicial script is a tight geoscript for which all cells are simplexes.

Definition 3.7. A geomap $G: \mathfrak{S} \rightarrow \mathfrak{S}^{\prime}$ between two tight geoscripts $\mathfrak{S}$ and $\mathfrak{S}^{\prime}$ is a collection of linear maps

$$
g_{k}: \mathfrak{S}_{k} \rightarrow \mathfrak{S}_{k}^{\prime}
$$

with the following two properties:

(a) the image of every $k$-surface $C_{k} \in \mathfrak{S}_{k}$ is a $k$-surface $C_{k}^{\prime} \in \mathfrak{S}_{k}^{\prime}$, e.g. on a $k-$ cell $c_{j}^{k}$ we have:

$$
g_{k}\left(c_{j}^{k}\right)=\sum \mu_{j}^{k, s} c_{s}^{\prime k}, \quad \mu_{j}^{k, s} \in\{-1,1\} .
$$


(b) for each $k$, the natural extension of $g_{k}$ to a set of $k$-chains fulfills the relation:

$$
\partial g_{k}\left(C_{k}\right)=g_{k-1}\left(\partial C_{k}\right)
$$

Moreover, $g_{k}$ is called tight if it maps tight surfaces to tight surfaces.

The notion of geomap can be used to define when two geoscripts are isomorphic. Let $\mathfrak{S}=\left\{\mathfrak{S}_{-1}, \mathfrak{S}_{0}, \mathfrak{S}_{1}, \ldots\right\}$ and $\mathfrak{T}=\left\{\mathfrak{T}_{-1}, \mathfrak{T}_{0}, \mathfrak{T}_{1}, \ldots\right\}$ be two geoscripts and suppose we have a geomap given by $g_{k}: \mathfrak{S}_{k} \rightarrow \mathfrak{C}(\mathfrak{T})_{k}$, from $\mathfrak{S}_{k}$ to the chains of $\mathfrak{T}_{k}$, that is such that for every cell $c_{j}^{k} \in \mathfrak{S}_{k}$,

$$
g_{k}\left(c_{j}^{k}\right)= \pm d_{j}^{k},
$$

where $d_{j}^{k} \in \mathfrak{T}_{k}$, i.e. suppose that $g_{k}$ is a bijection up to the sign between $\mathfrak{S}_{k}$ and $\mathfrak{T}_{k}$. Then we say that script $\mathfrak{S}$ is isomorphic to script $\mathfrak{T}$. It means essentially that one can change the signs of the cells provided one makes the necessary adjustments for the boundary map $\partial$, and these adjustments are determined by the relations $\partial g_{k}=g_{k-1} \partial$.

Definition 3.8. A geoscript $\mathfrak{S}^{\prime}$ is called a refinement of a given geoscript $\mathfrak{S}$ if there exists an injective geomap $G=\left\{g_{k}\right\}_{k}: \mathfrak{S} \rightarrow \mathfrak{S}^{\prime}$. A refinement is called tight if every $g_{k}$ is tight and if for each $k$, there exists only one surface $C_{k}^{\prime}$ inside the image $g_{k}\left(c_{j}^{k}\right)$ for which

$$
\partial C_{k}^{\prime}=\partial g_{k}\left(c_{j}^{k}\right)
$$

Theorem 3.9. Any tight geoscript $\mathfrak{S}$ admits a refinement to a simplicial script S.

Proof. The proof is done by induction over $k$, and it is left as an exercise for the avid reader.

We define the analog of the homology groups of a tight script $\mathfrak{S}$, due to the fact that the boundary operators $\partial: \mathfrak{C}_{k+1} \rightarrow \mathfrak{C}_{k}$ obey $\partial^{2}=0$ in all dimensions $k$. We define:

$$
\mathcal{H}_{k}(\mathfrak{S})=: Z_{k}(\mathfrak{S}) / B_{k}(\mathfrak{S})
$$

where $Z_{k}(\mathfrak{S})$ is the group of (closed oriented) $k$-cycles, and $B_{k}(\mathfrak{S})$ is the group of boundaries of $(k+1)$-chains of $\mathfrak{S}$.

Definition 3.10. We define the inner product of $k$-chains by

$$
\left\langle\sum_{s} \alpha_{s} c_{s}^{k}, \sum_{s} \beta_{s} c_{s}^{k}\right\rangle:=\sum \alpha_{s} \beta_{s}
$$

Then the exterior derivative $d$ on chains is defined by

$$
d c_{j}^{k}:=\sum_{\ell} \mu_{j}^{k, \ell} c_{\ell}^{k+1}
$$

naturally extended to the module of chains, and subject to the condition

$$
\left\langle d c_{j}^{k}, c_{\ell}^{k+1}\right\rangle=\left\langle c_{j}^{k}, \partial c_{\ell}^{k+1}\right\rangle .
$$


Similarly for the differential operators $d$, one can define the corresponding cohomology groups $\mathcal{H}^{k}(\mathfrak{S})$ of a tight script:

$$
\mathcal{H}^{k}(\mathfrak{S})=: Z^{k}(\mathfrak{S}) / B^{k}(\mathfrak{S})
$$

where $Z^{k}(\mathfrak{S})$ is the group of $(k+1)$-chains closed with respect to $d$, and $B^{k}(\mathfrak{S})$ is the group of coboundaries (in the image of $d$ ) of $k$-chains of $\mathfrak{S}$.

Note that in the case of a simplicial script $\mathfrak{S}$ being built by making use of usual (triangular) simplexes, it is similar to the usual notion of a simplicial complex. From dimension 3 and up a non-simplicial script does not uniquely determine the topology of the supporting space, though. Therefore, in a certain sense, scripts are a more loose concept than the traditional abstract simplexes.

\section{The discrete Dirac and Laplace operators on scripts}

Let $f$ be a function defined on a tight script $\mathfrak{S}$ with integer, real, complex, or Clifford algebra values. For example, if $\mathfrak{S}$ is has dimension $2, f$ is defined by

$$
\begin{aligned}
f & =f_{0}+f_{1}+f_{2}, \\
f_{0} & =\sum_{j \in \mathfrak{S}_{0}} f_{0 j} p_{j}, \quad f_{1}=\sum_{j \in \mathfrak{S}_{1}} f_{1 j} l_{j}, \quad f_{2}=\sum_{j \in \mathfrak{S}_{2}} f_{2 j} v_{j} .
\end{aligned}
$$

Definition 4.1. The discrete Hodge-Dirac operator for a tight script $\mathfrak{S}$ is defined as

$$
\not \partial=\partial+d
$$

acting on the corresponding parts of a function $f$.

For example, in the case $n=2$, we have:

$$
\begin{aligned}
\not \partial f & =\partial f_{1}+\left(d f_{0}+\partial f_{2}\right)+d f_{1} \\
& =\sum_{j \in \mathfrak{S}_{1}} f_{1 j} \partial l_{j}+\left(\sum_{j \in \mathfrak{S}_{0}} f_{0 j} d p_{j}+\sum_{j \in \mathfrak{S}_{2}} f_{2 j} \partial v_{j}\right)+\sum_{j \in \mathfrak{S}_{1}} f_{1 j} d l_{j}
\end{aligned}
$$

Definition 4.2. The discrete Laplace operator on a tight script $\mathfrak{S}$ is defined by:

$$
\Delta=\frac{1}{2}(\partial d+d \partial)=\frac{1}{2}(\partial+d)^{2}=\frac{1}{2} \not \partial^{2} .
$$

For example, for $f$ as above, we have:

$$
\begin{aligned}
2 \Delta f & =\partial\left(d f_{0}\right)+\left(\partial\left(d f_{1}\right)+d\left(\partial f_{1}\right)\right)+d\left(\partial f_{2}\right) \\
& =\sum_{j \in \mathfrak{S}_{0}} f_{0 j} \partial\left(d p_{j}\right)+\sum_{j \in \mathfrak{S}_{1}} f_{1 j}\left(\partial\left(d l_{j}\right)+d\left(\partial l_{j}\right)\right)+\sum_{j \in \mathfrak{S}_{2}} f_{2 j} d\left(\partial P_{j}\right) .
\end{aligned}
$$

Note that the Laplace operator defined above acts on all of $\mathfrak{S}$, not only on vertices (points). Let us remark that the above definition can be seen as a concretization of the abstract Hodge-Laplace operator [27]. Normally, the 
abstract definition is given in terms of the exterior derivative $d$ and its adjoint $d^{\star}$, but in our context we do not need to formally introduce the operator $d^{*}$. Furthermore, one of the requirements for the discretization of the abstract Hodge-Dirac operator in [27] is that the exterior derivative commutes with bounded (or smoothed) projections. This is not a trivial study and restricts their approach to simplicial complexes. While this is natural in the context of looking at simplicial decomposition of domains our setting is more general.

\section{Classic examples of scripts}

We give below concrete descriptions and computations of scripts obtained from classical examples of topological spaces.

\subsection{A Möbius strip}

As a topological space, the Möbius strip is obtained from a rectangle, identifying one pair of opposite edges in reverse orientation. In order to make it a tight geoscript, denoted by $\mathfrak{S}_{M}$, we obtain the same result by gluing two rectangles along one edge, as described in Figure 2. The script containes four

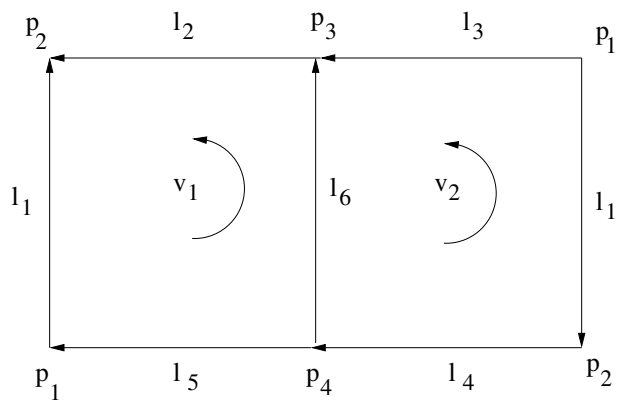

Figure 2. The Möbius script

points, six lines and two planes:

$$
\mathfrak{S}_{0}=\left\{p_{1}, p_{2}, p_{3}, p_{4}\right\}, \quad \mathfrak{S}_{1}=\left\{l_{1}, l_{2}, l_{3}, l_{4}, l_{5}, l_{6}\right\}, \quad \mathfrak{S}_{2}=\left\{v_{1}, v_{2}\right\} .
$$

The boundary operator $\partial$ acts on the Möbius script as follows:

$$
\begin{array}{llrl}
\partial l_{1}=p_{2}-p_{1}, & \partial l_{2}=p_{2}-p_{3}, \\
\partial l_{3}=p_{3}-p_{1}, & \partial l_{4}=p_{4}-p_{2}, \\
\partial l_{5}=p_{1}-p_{4}, & \partial l_{6}=p_{3}-p_{4} .
\end{array}
$$

Note that $\partial\left(l_{2}+l_{3}+l_{4}+l_{5}\right)=0$, so $l_{2}+l_{3}+l_{4}+l_{5}$ is a tight closed curve. Next, we have:

$$
\begin{aligned}
& \partial v_{1}=-l_{1}+l_{2}-l_{5}+l_{6}, \\
& \partial v_{2}=-l_{1}+l_{3}-l_{4}-l_{6} .
\end{aligned}
$$


Note that all linear combinations of the boundaries above have coefficients \pm 1 . Also, one can easily check that the boundary operator squares to 0 , as desired. For example:

$$
\begin{aligned}
\partial\left(\partial v_{1}\right) & =-\partial l_{1}+\partial l_{2}-\partial l_{5}+\partial l_{6} \\
& =-\left(p_{2}-p_{1}\right)+\left(p_{2}-p_{3}\right)-\left(p_{1}-p_{4}\right)+\left(p_{3}-p_{4}\right)=0 .
\end{aligned}
$$

Similarly $\partial\left(\partial v_{2}\right)=0$. Thefore, the Möbius script is a tight geoscript. Topologically is equivalent to a CW-complex consisting of one $2-$ cell: $\left(v_{1}+v_{2}\right)$, three 1-cells: $l_{1},\left(l_{2}+l_{3}\right),\left(l_{4}+l_{5}\right)$, and two $0-$ cells: $p_{1}, p_{2}$.

The script homology of $\mathfrak{S}_{M}$ is obtained in a similar fashion as one computes the homology of a CW-complex. In more detail, consider the sequence of chains:

$$
0 \stackrel{\partial}{\rightarrow} \mathfrak{C}_{2} \stackrel{\partial}{\rightarrow} \mathfrak{C}_{1} \stackrel{\partial}{\rightarrow} \mathfrak{C}_{0} \stackrel{\partial}{\rightarrow} 0
$$

We note that

$$
\partial\left(v_{1}+v_{2}\right)=-2 l_{1}+\left(l_{2}+l_{3}\right)-\left(l_{4}+l_{5}\right),
$$

so the image of the boundary of the sum of the two planes is non-empty. Its kernel is 0 , so $\mathcal{H}_{2}\left(\mathfrak{S}_{M}\right)=0$. Next,

$$
\partial l_{1}=p_{2}-p_{1}, \quad \partial\left(l_{2}+l_{3}\right)=p_{2}-p_{1}, \quad \partial\left(l_{4}+l_{5}\right)=p_{1}-p_{2},
$$

therefore up to a sign, $\left(l_{2}+l_{3}\right)-l_{1}$ and $\left(l_{4}+l_{5}\right)-l_{1}$ are homologous cycles. The kernel is two-dimensional (three line generators and the image is onedimensional), so isomorphic to $Z^{2}$. It follows that $\mathcal{H}_{1}\left(\mathfrak{S}_{M}\right)=\mathbb{Z}$. Similarly, one obtains $\mathcal{H}_{0}\left(\mathfrak{S}_{M}\right)=\mathbb{Z}$.

The differential operator $d$ acts on the script $\mathfrak{S}_{M}$ as follows:

$$
\begin{aligned}
& d p_{1}=-l_{1}-l_{3}+l_{5}, \\
& d p_{2}=l_{1}+l_{2}-l_{4}, \\
& d p_{3}=-l_{2}+l_{3}+l_{6}, \\
& d p_{4}=l_{4}-l_{5}-l_{6},
\end{aligned}
$$

and

$$
\begin{gathered}
d l_{1}=-v_{1}-v_{2}, \quad d l_{2}=v_{1}, \quad d l_{3}=v_{2}, \quad d l_{4}=-v_{2}, \\
d l_{5}=-v_{1}, \quad d l_{6}=v_{1}-v_{2} .
\end{gathered}
$$

For the cohomology of the script $\mathfrak{S}_{M}$, we study the sequence:

$$
0 \stackrel{d}{\rightarrow} \mathfrak{C}_{0} \stackrel{d}{\rightarrow} \mathfrak{C}_{1} \stackrel{d}{\rightarrow} \mathfrak{C}_{2} \stackrel{d}{\rightarrow} 0 .
$$

Note that $\sum_{j=1}^{4} d p_{j}=0$, therefore $\mathcal{H}^{0}\left(\mathfrak{S}_{M}\right)=\mathbb{Z}$. Because

$$
-d l_{1}=d\left(l_{2}+l_{3}\right)=-d\left(l_{4}+l_{5}\right)=v_{1}+v_{2},
$$

therefore $d\left(2 l_{1}+\left(l_{2}+l_{3}\right)-\left(l_{4}+l_{5}\right)\right)=0$, so the kernel of $d$ on lines is $3-2=1$ dimensional. Moreover, the image of $d$ on lines and the kernel of $d$ on planes 
are both generated by $v_{1}+v_{2}$. Summarizing, the script cohomology of the Möbius strip is indeed, as expected:

$$
\mathcal{H}^{0}\left(\mathfrak{S}_{M}\right)=\mathcal{H}^{1}\left(\mathfrak{S}_{M}\right)=\mathbb{Z}, \quad \mathcal{H}^{2}\left(\mathfrak{S}_{M}\right)=0 .
$$

Since the discrete Dirac operator is defined as $\not \partial=\partial+d$, using

$$
f=f_{0}+f_{1}+f_{2}, \quad f_{0}=\sum_{j=1}^{4} f_{0 j} p_{j}, \quad f_{1}=\sum_{j=1}^{6} f_{1 j} l_{j}, \quad f_{2}=\sum_{j=1}^{2} f_{2 j} v_{j},
$$

we have:

$$
\not \partial f=\partial f_{1}+\left(d f_{0}+\partial f_{2}\right)+d f_{1}
$$

Computations yield to:

$$
\partial f_{1}=\left[\begin{array}{cccccc}
-1 & 0 & -1 & 0 & 1 & 0 \\
1 & 1 & 0 & -1 & 0 & 0 \\
0 & -1 & 1 & 0 & 0 & 1 \\
0 & 0 & 0 & 1 & -1 & -1
\end{array}\right]\left[f_{1 j}\right]^{t}\left[p_{j}\right]
$$

where $\left[f_{1 j}\right]^{t}$ is the column vector of the corresponding 6 inputs, and $\left[p_{j}\right]$ is the row vector of the four points. Similarly we obtain:

$$
d f_{0}=\left[\begin{array}{cccc}
-1 & 1 & 0 & 0 \\
0 & 1 & -1 & 0 \\
-1 & 0 & 1 & 0 \\
0 & -1 & 0 & 1 \\
1 & 0 & 0 & -1 \\
0 & 0 & 1 & -1
\end{array}\right]\left[f_{0 j}\right]^{t}\left[l_{j}\right]
$$

where we notice that the matrix above is the transpose of the previous one for $\partial f_{1}$, as it should. Next we get:

$$
\partial f_{2}=\left[\begin{array}{cc}
-1 & -1 \\
1 & 0 \\
0 & 1 \\
0 & -1 \\
-1 & 0 \\
1 & -1
\end{array}\right]\left[f_{2 j}\right]^{t}\left[l_{j}\right]
$$

and

$$
d f_{1}=\left[\begin{array}{cccccc}
-1 & 1 & 0 & 0 & -1 & 1 \\
-1 & 0 & 1 & -1 & 0 & -1
\end{array}\right]\left[f_{1 j}\right]^{t}\left[v_{j}\right],
$$

Put together, the Dirac operator in matrix form is given as:

$$
\partial_{M}=\left(\begin{array}{cccc|ccccccccc}
0 & 0 & 0 & 0 & -1 & 0 & -1 & 0 & 1 & 0 & 0 & 0 \\
0 & 0 & 0 & 0 & 1 & 1 & 0 & -1 & 0 & 0 & 0 & 0 \\
0 & 0 & 0 & 0 & 0 & -1 & 1 & 0 & 0 & 1 & 0 & 0 \\
0 & 0 & 0 & 0 & 0 & 0 & 0 & 1 & -1 & -1 & 0 & 0 \\
\hline-1 & 1 & 0 & 0 & 0 & 0 & 0 & 0 & 0 & 0 & -1 & -1 \\
0 & 1 & -1 & 0 & 0 & 0 & 0 & 0 & 0 & 0 & 1 & 0 \\
-1 & 0 & 1 & 0 & 0 & 0 & 0 & 0 & 0 & 0 & 0 & 1 \\
0 & -1 & 0 & 1 & 0 & 0 & 0 & 0 & 0 & 0 & 0 & -1 \\
1 & 0 & 0 & -1 & 0 & 0 & 0 & 0 & 0 & 0 & -1 & 0 \\
0 & 0 & 1 & -1 & 0 & 0 & 0 & 0 & 0 & 0 & 1 & -1 \\
\hline 0 & 0 & 0 & 0 & -1 & 1 & 0 & 0 & -1 & 1 & 0 & 0 \\
0 & 0 & 0 & 0 & -1 & 0 & 1 & -1 & 0 & -1 & 0 & 0
\end{array}\right)
$$


with eigenvalues $-2,0,2$ of multiplicities $5,2,5$, respectively.

The discrete Laplace operator is given by:

$$
\begin{aligned}
2 \Delta f & =\partial\left(d f_{0}\right)+\left(\partial\left(d f_{1}\right)+d\left(\partial f_{1}\right)\right)+d\left(\partial f_{2}\right) \\
& =\sum_{j=0}^{3} f_{0 j} \partial\left(d p_{j}\right)+\sum_{j=1}^{8} f_{1 j}\left(\partial\left(d l_{j}\right)+d\left(\partial l_{j}\right)\right)+\sum_{j=1}^{4} f_{2 j} d\left(\partial v_{j}\right) .
\end{aligned}
$$

In matrix form we obtain:

$$
\begin{aligned}
2 \Delta_{M} f= & {\left[\begin{array}{cccc}
3 & -1 & -1 & -1 \\
-1 & 3 & -1 & -1 \\
-1 & -1 & 3 & -1 \\
-1 & -1 & -1 & 3
\end{array}\right]\left[f_{0 j}\right]^{t}\left[p_{j}\right] } \\
& +\left[\begin{array}{cccccc}
4 & 0 & 0 & 0 & 0 & 0 \\
0 & 3 & -1 & -1 & -1 & 0 \\
0 & -1 & 3 & -1 & -1 & 0 \\
0 & -1 & -1 & 3 & -1 & 0 \\
0 & -1 & -1 & -1 & 3 & 0 \\
0 & 0 & 0 & 0 & 0 & 4
\end{array}\right]\left[f_{1 j}\right]^{t}\left[l_{j}\right] \\
& +\left[\begin{array}{cc}
4 & 0 \\
0 & 4
\end{array}\right]\left[f_{2 j}\right]^{t}\left[v_{j}\right]
\end{aligned}
$$

In matrix form, the Laplacian of the Möbius script $\mathfrak{S}_{M}$ is given by the square of the Dirac matrix:

$$
\Delta_{M}=\frac{1}{2}\left(\begin{array}{cccc|cccccc|cc}
3 & -1 & -1 & -1 & 0 & 0 & 0 & 0 & 0 & 0 & 0 & 0 \\
-1 & 3 & -1 & -1 & 0 & 0 & 0 & 0 & 0 & 0 & 0 & 0 \\
-1 & -1 & 3 & -1 & 0 & 0 & 0 & 0 & 0 & 0 & 0 & 0 \\
-1 & -1 & -1 & 3 & 0 & 0 & 0 & 0 & 0 & 0 & 0 & 0 \\
\hline 0 & 0 & 0 & 0 & 4 & 0 & 0 & 0 & 0 & 0 & 0 & 0 \\
0 & 0 & 0 & 0 & 0 & 3 & -1 & -1 & -1 & 0 & 0 & 0 \\
0 & 0 & 0 & 0 & 0 & -1 & 3 & -1 & -1 & 0 & 0 & 0 \\
0 & 0 & 0 & 0 & 0 & -1 & -1 & 3 & -1 & 0 & 0 & 0 \\
0 & 0 & 0 & 0 & 0 & -1 & -1 & -1 & 3 & 0 & 0 & 0 \\
0 & 0 & 0 & 0 & 0 & 0 & 0 & 0 & 0 & 4 & 0 & 0 \\
\hline 0 & 0 & 0 & 0 & 0 & 0 & 0 & 0 & 0 & 0 & 4 & 0 \\
0 & 0 & 0 & 0 & 0 & 0 & 0 & 0 & 0 & 0 & 0 & 4
\end{array}\right) .
$$

\subsection{The Torus}

Consider the torus $T$ equipped with the script defined as in Figure 3. Topologically it is obtained by identifying the opposite sides of a rectangle with the same orientation. The boundary operator acts as follows:

$$
\begin{array}{ll}
\partial l_{1}=p_{1}-p_{0}, & \partial l_{2}=p_{0}-p_{1}, \\
\partial l_{3}=p_{0}-p_{2}, & \partial l_{4}=p_{2}-p_{0}, \\
\partial l_{5}=p_{3}-p_{2}, & \partial l_{6}=p_{2}-p_{3}, \\
\partial l_{7}=p_{1}-p_{3}, & \partial l_{8}=p_{3}-p_{1},
\end{array}
$$




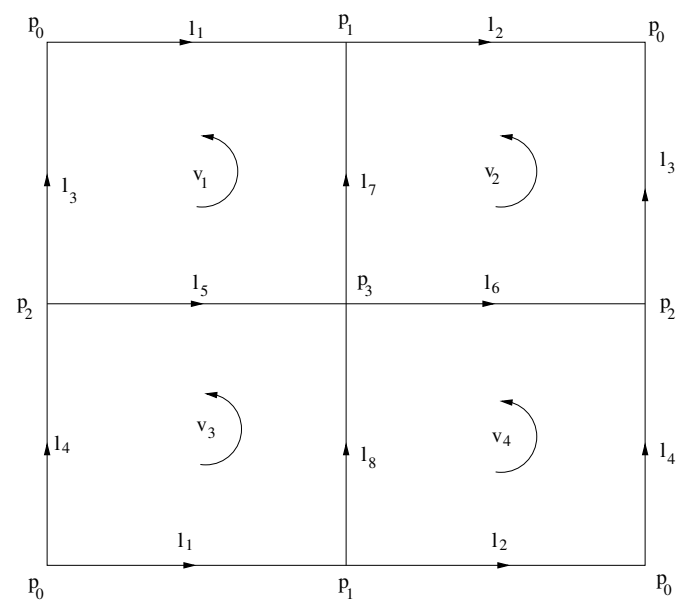

FiguRE 3. Torus script

and

$$
\begin{aligned}
& \partial v_{1}=l_{5}+l_{7}-l_{1}-l_{3}, \\
& \partial v_{2}=l_{6}+l_{3}-l_{2}-l_{7}, \\
& \partial v_{3}=l_{1}+l_{8}-l_{5}-l_{4}, \\
& \partial v_{4}=l_{2}+l_{4}-l_{6}-l_{8} .
\end{aligned}
$$

All 0,1 and 2-cells are tight cells, so the script $\mathfrak{S}_{T}$ above is a tight geoscript.

For the script homology of the torus, we consider the sequence of chains:

$$
0 \stackrel{\partial}{\rightarrow} \mathfrak{C}_{2} \stackrel{\partial}{\rightarrow} \mathfrak{C}_{1} \stackrel{\partial}{\rightarrow} \mathfrak{C}_{0} \stackrel{\partial}{\rightarrow} 0
$$

and we note that:

$$
\sum_{j=1}^{4} \partial l_{j}=0, \quad \sum_{j=1}^{4} \partial v_{j}=0,
$$

so, if we denote the line sums $l_{12}:=\left(l_{1}+l_{2}\right)$ and $l_{34}:=\left(l_{3}+l_{4}\right)$, and $\gamma$ the sum of all $v_{j}$, we have:

$$
\partial\left(l_{12}\right)=\partial\left(l_{34}\right)=0, \quad \partial \gamma=0
$$

It turns out that $l_{12}$ and $l_{34}$ are a basis of $\mathcal{H}_{1}\left(\mathfrak{S}_{T}\right)$ and $\gamma$ is the generator of $\mathcal{H}_{2}\left(\mathfrak{S}_{T}\right)$, i.e. we capture the script homology of the torus:

$$
\mathcal{H}_{0}\left(\mathfrak{S}_{T}\right)=\mathbb{Z}, \quad \mathcal{H}_{1}\left(\mathfrak{S}_{T}\right)=\mathbb{Z} \oplus \mathbb{Z}, \quad \mathcal{H}_{2}\left(\mathfrak{S}_{T}\right)=\mathbb{Z} .
$$

At the differential operator level, we obtain:

$$
\begin{aligned}
& d p_{0}=-l_{1}+l_{2}+l_{3}-l_{4}, \\
& d p_{1}=l_{1}-l_{2}+l_{7}-l_{8}, \\
& d p_{2}=-l_{3}+l_{4}-l_{5}+l_{6}, \\
& d p_{3}=l_{5}-l_{6}-l_{7}+l_{8},
\end{aligned}
$$


and

$$
\begin{aligned}
d l_{1}=-v_{1}+v_{3}, & d l_{2}=-v_{2}+v_{4}, \\
d l_{3}=-v_{1}+v_{2}, & d l_{4}=-v_{3}+v_{4}, \\
d l_{5}=v_{1}-v_{3}, & d l_{6}=-v_{4}+v_{2}, \\
d l_{7}=v_{1}-v_{2}, & d l_{8}=v_{3}-v_{4} .
\end{aligned}
$$

Note that $\sum_{j=0}^{3} d p_{j}=0, \sum_{j=1}^{8} l_{j}=0$, and using the notation for the sum of two lines, $l_{i j}:=l_{i}+l_{j}$, we have:

$$
\begin{array}{ll}
d\left(l_{15}-l_{26}\right)=0, & d\left(p_{0}+p_{2}\right)=-d\left(p_{1}+p_{3}\right)=-\left(l_{15}-l_{26}\right), \\
d\left(l_{37}-l_{48}\right)=0, & d\left(p_{0}+p_{1}\right)=-d\left(p_{2}+p_{3}\right)=\left(l_{37}-l_{48}\right) .
\end{array}
$$

Therefore each $\mathcal{H}^{0}\left(\mathfrak{S}_{T}\right)$ and $\mathcal{H}^{2}\left(\mathfrak{S}_{T}\right)$ have one generator, and $\mathcal{H}^{1}\left(\mathfrak{S}_{T}\right)$ has two generators. Summarazing, we obtain the script cohomology groups of $\mathfrak{S}_{T}$ is given by:

$$
\mathcal{H}^{0}\left(\mathfrak{S}_{T}\right)=\mathbb{Z}, \quad \mathcal{H}^{1}\left(\mathfrak{S}_{T}\right)=\mathbb{Z} \oplus \mathbb{Z}, \quad \mathcal{H}^{2}\left(\mathfrak{S}_{T}\right)=\mathbb{Z} .
$$

Since the discrete Dirac operator is defined as $\not \partial=\partial+d$, using

$$
f=f_{0}+f_{1}+f_{2}, \quad f_{0}=\sum_{j=0}^{3} f_{0 j} p_{j}, \quad f_{1}=\sum_{j=1}^{8} f_{1 j} l_{j}, \quad f_{2}=\sum_{j=1}^{4} f_{2 j} v_{j},
$$

we have:

$$
\not \partial f=\partial f_{1}+\left(d f_{0}+\partial f_{2}\right)+d f_{1} .
$$

Computations yield to:

$$
\partial f_{1}=\left[\begin{array}{cccccccc}
-1 & 1 & 1 & -1 & 0 & 0 & 0 & 0 \\
1 & -1 & 0 & 0 & 0 & 0 & 1 & -1 \\
0 & 0 & -1 & 1 & -1 & 1 & 0 & 0 \\
0 & 0 & 0 & 0 & 1 & -1 & -1 & 1
\end{array}\right]\left[f_{1 j}\right]^{t}\left[p_{j}\right]
$$

where $\left[f_{1 j}\right]^{t}$ is the column vector the corresponding 8 inputs, and $\left[p_{j}\right]$ is the row vector of the four points. Similarly we obtain:

$$
d f_{0}=\left[\begin{array}{cccc}
-1 & 1 & 0 & 0 \\
1 & -1 & 0 & 0 \\
1 & 0 & -1 & 0 \\
-1 & 0 & 1 & 0 \\
0 & 0 & -1 & 1 \\
0 & 0 & 1 & -1 \\
0 & 1 & 0 & -1 \\
0 & -1 & 0 & 1
\end{array}\right]\left[f_{0 j}\right]^{t}\left[l_{j}\right]
$$


where we notice that the matrix above is the transpose of the previous one for $\partial f_{1}$. Next we get:

$$
\partial f_{2}=\left[\begin{array}{cccc}
-1 & 0 & 1 & 0 \\
0 & -1 & 0 & 1 \\
-1 & 1 & 0 & 0 \\
0 & 0 & -1 & 1 \\
1 & 0 & -1 & 0 \\
0 & 1 & 0 & -1 \\
1 & -1 & 0 & 0 \\
0 & 0 & 1 & -1
\end{array}\right]\left[f_{2 j}\right]^{t}\left[l_{j}\right]
$$

and

$$
d f_{1}=\left[\begin{array}{cccccccc}
-1 & 0 & -1 & 0 & 1 & 0 & 1 & 0 \\
0 & -1 & 1 & 0 & 0 & 1 & -1 & 0 \\
1 & 0 & 0 & -1 & -1 & 0 & 0 & 1 \\
0 & 1 & 0 & 1 & 0 & -1 & 0 & -1
\end{array}\right]\left[f_{1 j}\right]^{t}\left[v_{j}\right]
$$

Put together, the Dirac operator on the torus script in matrix form is given

\begin{tabular}{|c|c|c|c|c|c|c|c|c|c|c|c|c|c|c|c|}
\hline 0 & 0 & 0 & 0 & -1 & 1 & 1 & -1 & 0 & 0 & 0 & 0 & 0 & 0 & 0 & 0 \\
\hline 0 & 0 & 0 & 0 & 1 & -1 & 0 & 0 & 0 & 0 & 1 & -1 & 0 & 0 & 0 & 0 \\
\hline 0 & 0 & 0 & 0 & 0 & 0 & -1 & 1 & -1 & 1 & 0 & 0 & 0 & 0 & 0 & 0 \\
\hline 0 & 0 & 0 & 0 & 0 & 0 & 0 & 0 & 1 & -1 & -1 & 1 & 0 & 0 & 0 & 0 \\
\hline-1 & 1 & 0 & 0 & 0 & 0 & 0 & 0 & 0 & 0 & 0 & 0 & -1 & 0 & 1 & 0 \\
\hline 1 & -1 & 0 & 0 & 0 & 0 & 0 & 0 & 0 & 0 & 0 & 0 & 0 & -1 & 0 & 1 \\
\hline 1 & 0 & -1 & 0 & 0 & 0 & 0 & 0 & 0 & 0 & 0 & 0 & -1 & 1 & 0 & 0 \\
\hline-1 & 0 & 1 & 0 & 0 & 0 & 0 & 0 & 0 & 0 & 0 & 0 & 0 & 0 & -1 & 1 \\
\hline 0 & 0 & -1 & 1 & 0 & 0 & 0 & 0 & 0 & 0 & 0 & 0 & 1 & 0 & -1 & 0 \\
\hline 0 & 0 & 1 & -1 & 0 & 0 & 0 & 0 & 0 & 0 & 0 & 0 & 0 & 1 & 0 & -1 \\
\hline 0 & 1 & 0 & -1 & 0 & 0 & 0 & 0 & 0 & 0 & 0 & 0 & 1 & -1 & 0 & 0 \\
\hline 0 & -1 & 0 & 1 & 0 & 0 & 0 & 0 & 0 & 0 & 0 & 0 & 0 & 0 & 1 & -1 \\
\hline 0 & 0 & 0 & 0 & -1 & 0 & -1 & 0 & 1 & 0 & 1 & 0 & 0 & 0 & 0 & 0 \\
\hline 0 & 0 & 0 & 0 & 0 & -1 & 1 & 0 & 0 & 1 & -1 & 0 & 0 & 0 & 0 & 0 \\
\hline 0 & 0 & 0 & 0 & 1 & 0 & 0 & -1 & -1 & 0 & 0 & 1 & 0 & 0 & 0 & 0 \\
\hline 0 & 0 & 0 & 0 & 0 & 1 & 0 & 1 & 0 & -1 & 0 & -1 & 0 & 0 & 0 & 0 \\
\hline
\end{tabular}
as:

with eigenvalues $-2 \sqrt{2},-2,0,2,2 \sqrt{2}$ of multiplicities $2,4,4,4,2$, respectively.

The discrete Laplace operator is given by:

$$
\begin{aligned}
2 \Delta_{T} f & =\partial\left(d f_{0}\right)+\left(\partial\left(d f_{1}\right)+d\left(\partial f_{1}\right)\right)+d\left(\partial f_{2}\right) \\
& =\sum_{j=0}^{3} f_{0 j} \partial\left(d p_{j}\right)+\sum_{j=1}^{8} f_{1 j}\left(\partial\left(d l_{j}\right)+d\left(\partial l_{j}\right)\right)+\sum_{j=1}^{4} f_{2 j} d\left(\partial v_{j}\right) .
\end{aligned}
$$


We obtain:

$$
\begin{aligned}
& \Delta_{T} f=\left[\begin{array}{cccc}
2 & -1 & -1 & 0 \\
-1 & 2 & 0 & -1 \\
-1 & 0 & 2 & -1 \\
0 & -1 & -1 & 2
\end{array}\right]\left[f_{0 j}\right]^{t}\left[p_{j}\right] \\
& +\left[\begin{array}{cccccccc}
2 & -1 & 0 & 0 & -1 & 0 & 0 & 0 \\
-1 & 2 & 0 & 0 & 0 & -1 & 0 & 0 \\
0 & 0 & 2 & -1 & 0 & 0 & -1 & 0 \\
0 & 0 & -1 & 2 & 0 & 0 & 0 & -1 \\
-1 & 0 & 0 & 0 & 2 & -1 & 0 & 0 \\
0 & -1 & 0 & 0 & -1 & 2 & 0 & 0 \\
0 & 0 & -1 & 0 & 0 & 0 & 2 & -1 \\
0 & 0 & 0 & -1 & 0 & 0 & -1 & 2
\end{array}\right]\left[f_{1 j}\right]^{t}\left[l_{j}\right] \\
& +\left[\begin{array}{cccc}
2 & -1 & -1 & 0 \\
-1 & 2 & 0 & -1 \\
-1 & 0 & 2 & -1 \\
0 & -1 & -1 & 2
\end{array}\right]\left[f_{2 j}\right]^{t}\left[v_{j}\right]
\end{aligned}
$$

\begin{tabular}{|c|c|c|c|c|c|c|c|c|c|c|c|c|c|c|c|}
\hline 2 & -1 & -1 & 0 & 0 & 0 & 0 & 0 & 0 & 0 & 0 & 0 & 0 & 0 & 0 & 0 \\
\hline-1 & 2 & 0 & -1 & 0 & 0 & 0 & 0 & 0 & 0 & 0 & 0 & 0 & 0 & 0 & 0 \\
\hline-1 & 0 & 2 & -1 & 0 & 0 & 0 & 0 & 0 & 0 & 0 & 0 & 0 & 0 & 0 & 0 \\
\hline 0 & -1 & -1 & 2 & 0 & 0 & 0 & 0 & 0 & 0 & 0 & 0 & 0 & 0 & 0 & 0 \\
\hline 0 & 0 & 0 & 0 & 2 & -1 & 0 & 0 & -1 & 0 & 0 & 0 & 0 & 0 & 0 & 0 \\
\hline 0 & 0 & 0 & 0 & -1 & 2 & 0 & 0 & 0 & -1 & 0 & 0 & 0 & 0 & 0 & 0 \\
\hline 0 & 0 & 0 & 0 & 0 & 0 & 2 & -1 & 0 & 0 & -1 & 0 & 0 & 0 & 0 & 0 \\
\hline 0 & 0 & 0 & 0 & 0 & 0 & -1 & 2 & 0 & 0 & 0 & -1 & 0 & 0 & 0 & 0 \\
\hline 0 & 0 & 0 & 0 & -1 & 0 & 0 & 0 & 2 & -1 & 0 & 0 & 0 & 0 & 0 & 0 \\
\hline 0 & 0 & 0 & 0 & 0 & -1 & 0 & 0 & -1 & 2 & 0 & 0 & 0 & 0 & 0 & 0 \\
\hline 0 & 0 & 0 & 0 & 0 & 0 & -1 & 0 & 0 & 0 & 2 & -1 & 0 & 0 & 0 & 0 \\
\hline 0 & 0 & 0 & 0 & 0 & 0 & 0 & -1 & 0 & 0 & -1 & 2 & 0 & 0 & 0 & 0 \\
\hline 0 & 0 & 0 & 0 & 0 & 0 & 0 & 0 & 0 & 0 & 0 & 0 & 2 & -1 & -1 & 0 \\
\hline 0 & 0 & 0 & 0 & 0 & 0 & 0 & 0 & 0 & 0 & 0 & 0 & -1 & 2 & 0 & -1 \\
\hline 0 & 0 & 0 & 0 & 0 & 0 & 0 & 0 & 0 & 0 & 0 & 0 & -1 & 0 & 2 & -1 \\
\hline 0 & 0 & 0 & 0 & 0 & 0 & 0 & 0 & 0 & 0 & 0 & 0 & 0 & -1 & -1 & 2 \\
\hline
\end{tabular}

In matrix form, the Laplacian of the script for the torus $T$ is given by the square of the Dirac matrix:

\subsection{The Klein bottle}

Consider the Klein bottle equipped with the script $\mathfrak{S}_{K}$ defined as in Figure 4 . It is obtained from a rectangle identifying one pair of opposite sides with the same orientations, and the other pair is identified with opposite line orientations. We obtain the following script geometry of the Klein script:

$$
\begin{array}{ll}
\partial l_{1}=p_{1}-p_{0}, & \partial l_{2}=p_{0}-p_{1}, \\
\partial l_{3}=p_{2}-p_{0}, & \partial l_{4}=p_{0}-p_{2}, \\
\partial l_{5}=p_{3}-p_{2}, & \partial l_{6}=p_{2}-p_{3}, \\
\partial l_{7}=p_{1}-p_{3}, & \partial l_{4}=p_{3}-p_{1},
\end{array}
$$




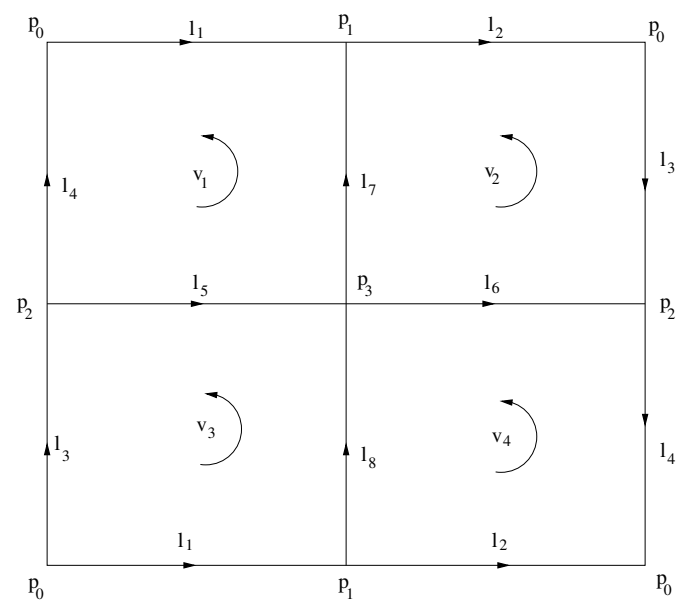

FiguRE 4. Klein script

and

$$
\begin{aligned}
& \partial v_{1}=l_{5}+l_{7}-l_{1}-l_{4}, \\
& \partial v_{2}=l_{6}-l_{3}-l_{2}-l_{7}, \\
& \partial v_{3}=l_{1}+l_{8}-l_{5}-l_{3}, \\
& \partial v_{4}=l_{2}-l_{4}-l_{6}-l_{8} .
\end{aligned}
$$

Note again that this is a tight script, and we have:

$$
\sum_{j=1}^{4} \partial v_{j}=-2\left(l_{3}+l_{4}\right) \text {. }
$$

If we denote the line sum $l_{12}:=\left(l_{1}+l_{2}\right)$ and $l_{34}:=\left(l_{3}+l_{4}\right)$, and $\gamma$ is the sum of all $v_{j}$, then $l_{12}$ is a generator for $\mathcal{H}_{1}\left(\mathfrak{S}_{K}\right)$ modulo torsion, and $l_{34}$ is a torsion element of $\mathcal{H}_{1}\left(\mathfrak{S}_{K}\right)$. But $\gamma$ is not a cycle anymore, as $\partial \gamma=-2 l_{34}$, i.e. we recapture the script homology of the Klein bottle:

$$
\mathcal{H}_{0}\left(\mathfrak{S}_{K}\right)=\mathbb{Z}, \quad \mathcal{H}_{1}\left(\mathfrak{S}_{K}\right)=\mathbb{Z} \oplus \mathbb{Z}_{2}, \quad \mathcal{H}_{2}\left(\mathfrak{S}_{K}\right)=0
$$

At the differential operator level, we obtain:

$$
\begin{aligned}
& d p_{0}=-l_{1}+l_{2}-l_{3}+l_{4}, \\
& d p_{1}=l_{1}-l_{2}+l_{7}-l_{8}, \\
& d p_{2}=l_{3}-l_{4}-l_{5}+l_{6}, \\
& d p_{3}=l_{5}-l_{6}-l_{7}+l_{8},
\end{aligned}
$$


and

$$
\begin{aligned}
d l_{1}=-v_{1}+v_{3}, & d l_{2}=-v_{2}+v_{4}, \\
d l_{3}=-v_{2}-v_{3}, & d l_{4}=-v_{1}-v_{4}, \\
d l_{5}=v_{1}-v_{3}, & d l_{6}=-v_{4}+v_{2}, \\
d l_{7}=v_{1}-v_{2}, & d l_{8}=v_{3}-v_{4} .
\end{aligned}
$$

Note that $\sum_{j=0}^{3} d p_{j}=0$ and $d\left(\left(l_{1}-l_{2}\right)+\left(l_{3}-l_{4}\right)\right)=0$. Therefore $\mathcal{H}^{1}\left(\mathfrak{S}_{K}\right)$ is generated by one element. Next, we have:

$$
d l_{12}=-\left(v_{1}+v_{2}\right)+\left(v_{3}+v_{4}\right), \quad d l_{34}=-\left(v_{1}+v_{2}\right)-\left(v_{3}+v_{4}\right),
$$

therefore, because $d\left(l_{12}+l_{34}\right)=-2\left(v_{1}+v_{2}\right)$, we obtain that $\mathcal{H}^{2}\left(\mathfrak{S}_{K}\right)=\mathbb{Z}_{2}$. This yields the script cohomology groups of $\mathfrak{S}_{K}$ :

$$
\mathcal{H}^{0}\left(\mathfrak{S}_{K}\right)=\mathbb{Z}, \quad \mathcal{H}^{1}\left(\mathfrak{S}_{K}\right)=\mathbb{Z}, \quad \mathcal{H}^{2}\left(\mathfrak{S}_{K}\right)=\mathbb{Z}_{2}
$$

The discrete Dirac operator for the Klein script above is given by the formula:

$$
\not \partial f=\partial f_{1}+\left(d f_{0}+\partial f_{2}\right)+d f_{1}
$$

where

$$
f=f_{0}+f_{1}+f_{2}, \quad f_{0}=\sum_{j=0}^{3} f_{0 j} p_{j}, \quad f_{1}=\sum_{j=1}^{8} f_{1 j} l_{j}, \quad f_{2}=\sum_{j=1}^{4} f_{2 j} v_{j} .
$$

Computations yield the following results:

$$
\begin{gathered}
\partial f_{1}=\left[\begin{array}{cccccccc}
-1 & 1 & -1 & 1 & 0 & 0 & 0 & 0 \\
1 & -1 & 0 & 0 & 0 & 0 & 1 & -1 \\
0 & 0 & 1 & -1 & -1 & 1 & 0 & 0 \\
0 & 0 & 0 & 0 & 1 & -1 & -1 & 1
\end{array}\right]\left[f_{1 j}\right]^{t}\left[p_{j}\right] \\
d f_{0}=\left[\begin{array}{cccc}
-1 & 1 & 0 & 0 \\
1 & -1 & 0 & 0 \\
-1 & 0 & 1 & 0 \\
1 & 0 & -1 & 0 \\
0 & 0 & -1 & 1 \\
0 & 0 & 1 & -1 \\
0 & 1 & 0 & -1 \\
0 & -1 & 0 & 1
\end{array}\right]\left[f_{0 j}\right]^{t}\left[l_{j}\right] .
\end{gathered}
$$


Next we get:

$$
\partial f_{2}=\left[\begin{array}{cccc}
-1 & 0 & 1 & 0 \\
0 & -1 & 0 & 1 \\
0 & -1 & -1 & 0 \\
-1 & 0 & 0 & -1 \\
1 & 0 & -1 & 0 \\
0 & 1 & 0 & -1 \\
1 & -1 & 0 & 0 \\
0 & 0 & 1 & -1
\end{array}\right]\left[f_{2 j}\right]^{t}\left[l_{j}\right]
$$

and

$$
d f_{1}=\left[\begin{array}{cccccccc}
-1 & 0 & 0 & -1 & 1 & 0 & 1 & 0 \\
0 & -1 & -1 & 0 & 0 & 1 & -1 & 0 \\
1 & 0 & -1 & 0 & -1 & 0 & 0 & 1 \\
0 & 1 & 0 & -1 & 0 & -1 & 0 & -1
\end{array}\right]\left[f_{1 j}\right]^{t}\left[v_{j}\right]
$$

In matrix form, the Dirac operator for the Klein script is:

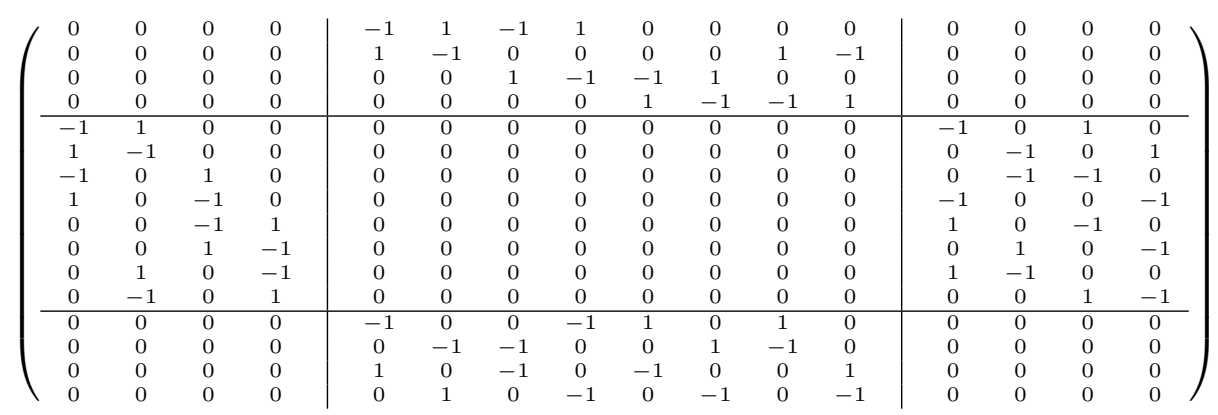

with eigenvalues $-2 \sqrt{2},-2,-\sqrt{2}, 0, \sqrt{2}, 2,2 \sqrt{2}$ of multiplicites $2,3,2,2,2,3,2$, respectively. 
The Laplace operator is given by:

$$
\begin{aligned}
\Delta_{K} f= & {\left[\begin{array}{cccc}
2 & -1 & -1 & 0 \\
-1 & 2 & 0 & -1 \\
-1 & 0 & 2 & -1 \\
0 & -1 & -1 & 2
\end{array}\right]\left[f_{0 j}\right]^{t}\left[p_{j}\right] } \\
& +\left[\begin{array}{cccccccc}
2 & -1 & 0 & 0 & -1 & 0 & 0 & 0 \\
-1 & 2 & 0 & 0 & 0 & -1 & 0 & 0 \\
0 & 0 & 2 & -1 & 0 & 0 & 1 & -1 \\
0 & 0 & -1 & 2 & 0 & 0 & -1 & 1 \\
-1 & 0 & 0 & 0 & 2 & -1 & 0 & 0 \\
0 & -1 & 0 & 0 & -1 & 2 & 0 & 0 \\
0 & 0 & 1 & -1 & 0 & 0 & 2 & -1 \\
0 & 0 & -1 & 1 & 0 & 0 & -1 & 2
\end{array}\right]\left[f_{1 j}\right]^{t}\left[l_{j}\right] \\
& +\frac{1}{2}\left[\begin{array}{cccccc}
4 & -1 & -2 & 1 & & \\
-1 & 4 & 1 & -2 \\
-2 & 1 & 4 & -1 \\
1 & -2 & -1 & 4
\end{array}\right]\left[f_{2 j}\right]^{t}\left[v_{j}\right] .
\end{aligned}
$$

\subsection{The Real Projective Plane}

We investigate several scripts for the projective plane $\mathbb{R P}^{2}$. The simplest one is given in Figure 5, but it is not a geoscript. Indeed, this script, denoted by

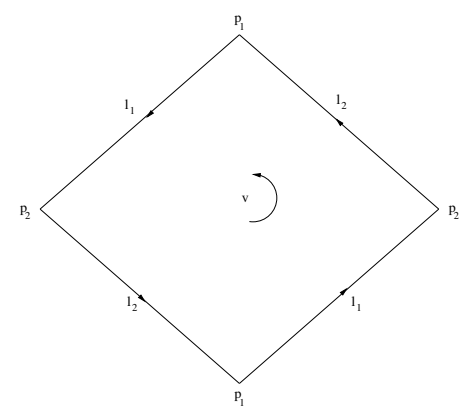

FIGURE 5. Simplest projective script

$\mathfrak{S}_{\mathbb{R}^{2}, 1}$ is characterized by

$$
\begin{array}{llrl}
\mathfrak{S}_{0}=\left\{p_{1}, p_{2}\right\}, & \mathfrak{S}_{1}=\left\{l_{1}, l_{2}\right\}, & \mathfrak{S}_{2}=\{v\}, \\
\partial l_{1}=p_{2}-p_{1}, & \partial l_{2}=p_{1}-p_{2}, & \partial v=2 l_{1}+2 l_{2} .
\end{array}
$$

Therefore the boundary of the $2-$ chain $v$ is not a geochain, as it contains coefficients different than \pm 1 .

Denoting the line sum $l_{12}:=\left(l_{1}+l_{2}\right)$ then $l_{12}$ is a representing the nonzero element of $\mathcal{H}_{1}\left(\mathfrak{S}_{\mathbb{R}^{2}}\right)$, and $v$ is not a cycle, as $\partial v=2 l_{12}$, i.e. we obtain 
the script homology of the $\mathbb{R P}^{2}$ :

$$
\mathcal{H}_{0}\left(\mathfrak{S}_{\mathbb{R} \mathbb{P}^{2}}\right)=\mathbb{Z}, \quad \mathcal{H}_{1}\left(\mathfrak{S}_{\mathbb{R}^{2}}\right)=\mathbb{Z}_{2}, \quad \mathcal{H}_{2}\left(\mathfrak{S}_{\mathbb{R}^{2}}\right)=0
$$

For the differential operator $d$ we get:

$$
\begin{gathered}
d p_{1}=-\left(l_{1}-l_{2}\right), \quad d p_{2}=l_{1}-l_{2}, \\
d l_{1}=d l_{2}=v
\end{gathered}
$$

Note that the kernel of $d$ on points is generated by $p_{1}+p_{2}$, so $\mathcal{H}^{0}\left(\mathfrak{S}_{\mathbb{R P}}\right)=\mathbb{Z}$. Next, the kernel of $d$ on lines and the image of $d$ on points are both generated by $l_{1}-l_{2}$, which yields to $\mathcal{H}^{1}\left(\mathfrak{S}_{\mathbb{R}^{2}}\right)=0$. The image of $d\left(l_{1}+l_{2}\right)=2 v$, therefore $\mathcal{H}^{2}\left(\mathfrak{S}_{\mathbb{R P}^{2}}\right)=\mathbb{Z}_{2}$.

We compute the Dirac and Laplace operators on this script, yielding:

$$
\partial_{\mathbb{R P}^{2}}=\left(\begin{array}{cc|cc|c}
0 & 0 & -1 & 1 & 0 \\
0 & 0 & 1 & -1 & 0 \\
\hline-1 & 1 & 0 & 0 & 2 \\
1 & -1 & 0 & 0 & 2 \\
\hline 0 & 0 & 2 & -2 & 0
\end{array}\right)
$$

with eigenvalues $-2 \sqrt{2},-2,0,2,2 \sqrt{2}$, all having multiplicities 1 .

The Laplacian operator for this script depicting $\mathbb{R P}^{2}$ is:

$$
\Delta_{\mathbb{R P}^{2}}=\frac{1}{2}\left(\begin{array}{cc|cc|c}
1 & -1 & 0 & 0 & 0 \\
-1 & 1 & 0 & 0 & 0 \\
\hline 0 & 0 & 3 & 1 & 0 \\
0 & 0 & 1 & 3 & 0 \\
\hline 0 & 0 & 0 & 0 & 4
\end{array}\right) .
$$

In order to obtain a tight geoscript for the projective plane, we add one more point $p_{0}$ and four lines to the script above, as in Figure 6. We obtain:

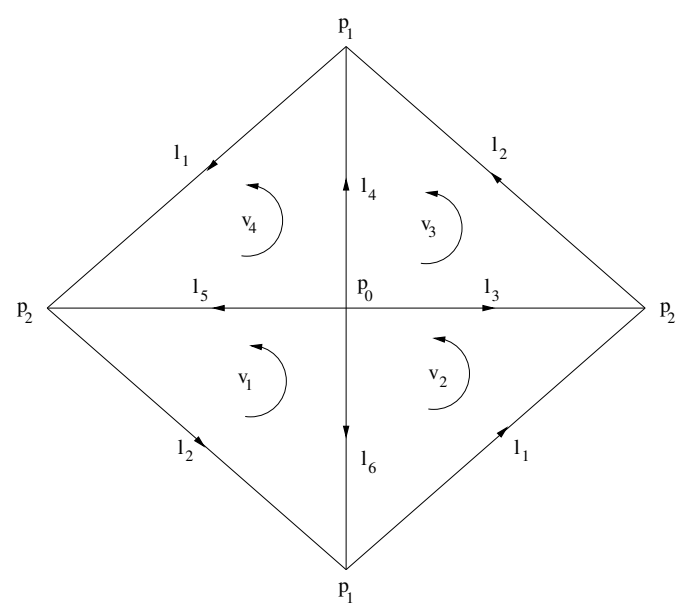

Figure 6. A tight geoscript for $\mathbb{R P}^{2}$ 


$$
\begin{array}{ll}
\partial l_{1}=p_{2}-p_{1}, & \partial l_{2}=p_{1}-p_{2}, \\
\partial l_{3}=p_{2}-p_{0}, & \partial l_{4}=p_{1}-p_{0}, \\
\partial l_{5}=p_{2}-p_{0}, & \partial l_{6}=p_{1}-p_{0},
\end{array}
$$

and

$$
\begin{aligned}
& \partial v_{1}=l_{2}-l_{6}+l_{5}, \\
& \partial v_{2}=l_{1}-l_{3}+l_{6}, \\
& \partial v_{3}=l_{2}-l_{4}+l_{3}, \\
& \partial v_{4}=l_{1}-l_{5}+l_{4} .
\end{aligned}
$$

At the differential operator level, we obtain:

$$
\begin{aligned}
& d p_{0}=-l_{3}-l_{4}-l_{5}-l_{6}, \\
& d p_{1}=-l_{1}+l_{2}+l_{4}+l_{6}, \\
& d p_{2}=l_{1}-l_{2}+l_{3}+l_{5},
\end{aligned}
$$

and

$$
\begin{aligned}
d l_{1}=v_{2}+v_{4}, & d l_{2}=v_{1}+v_{3}, \\
d l_{3}=-v_{2}+v_{3}, & d l_{4}=-v_{3}+v_{4}, \\
d l_{5}=v_{1}-v_{4}, & d l_{6}=v_{2}-v_{1} .
\end{aligned}
$$

The homology and cohomology of this real projective plane script is computed in a similar way as in the case of the first $\mathbb{R P}^{2}$ script, yielding, of course, the same result.

The discrete Dirac operator for the script above is given by:

$$
\not \partial f=\partial f_{1}+\left(d f_{0}+\partial f_{2}\right)+d f_{1}
$$

where

$$
f=f_{0}+f_{1}+f_{2}, \quad f_{0}=\sum_{j=0}^{2} f_{0 j} p_{j}, \quad f_{1}=\sum_{j=1}^{6} f_{1 j} l_{j}, \quad f_{2}=\sum_{j=1}^{4} f_{2 j} v_{j} .
$$

Computations yield the following results:

$$
\begin{gathered}
\partial f_{1}=\left[\begin{array}{cccccc}
0 & 0 & -1 & -1 & -1 & -1 \\
-1 & 1 & 0 & 1 & 0 & 1 \\
1 & -1 & 1 & 0 & 1 & 0
\end{array}\right]\left[f_{1 j}\right]^{t}\left[p_{j}\right] \\
d f_{0}=\left[\begin{array}{ccc}
0 & -1 & 1 \\
0 & 1 & -1 \\
-1 & 0 & 1 \\
-1 & 1 & 0 \\
-1 & 0 & 1 \\
-1 & 1 & 0
\end{array}\right]\left[f_{0 j}\right]^{t}\left[l_{j}\right]
\end{gathered}
$$




$$
\partial f_{2}=\left[\begin{array}{cccc}
0 & 1 & 0 & 1 \\
1 & 0 & 1 & 0 \\
0 & -1 & 1 & 0 \\
0 & 0 & -1 & 1 \\
1 & 0 & 0 & -1 \\
-1 & 1 & 0 & 0
\end{array}\right]\left[f_{2 j}\right]^{t}\left[l_{j}\right]
$$

and

$$
d f_{1}=\left[\begin{array}{cccccc}
0 & 1 & 0 & 0 & 1 & -1 \\
1 & 0 & -1 & 0 & 0 & 1 \\
0 & 1 & 1 & -1 & 0 & 0 \\
1 & 0 & 0 & 1 & -1 & 0
\end{array}\right]\left[f_{1 j}\right]^{t}\left[v_{j}\right]
$$

In full matrix form we obtain

$\left(\begin{array}{ccc|cccccc|cccc}0 & 0 & 0 & 0 & 0 & -1 & -1 & -1 & -1 & 0 & 0 & 0 & 0 \\ 0 & 0 & 0 & -1 & 1 & 0 & 1 & 0 & 1 & 0 & 0 & 0 & 0 \\ 0 & 0 & 0 & 1 & -1 & 1 & 0 & 1 & 0 & 0 & 0 & 0 & 0 \\ \hline 0 & -1 & 1 & 0 & 0 & 0 & 0 & 0 & 0 & 0 & 1 & 0 & 1 \\ 0 & 1 & -1 & 0 & 0 & 0 & 0 & 0 & 0 & 1 & 0 & 1 & 0 \\ -1 & 0 & 1 & 0 & 0 & 0 & 0 & 0 & 0 & 0 & -1 & 1 & 0 \\ -1 & 1 & 0 & 0 & 0 & 0 & 0 & 0 & 0 & 0 & 0 & -1 & 1 \\ -1 & 0 & 1 & 0 & 0 & 0 & 0 & 0 & 0 & 1 & 0 & 0 & -1 \\ -1 & 1 & 0 & 0 & 0 & 0 & 0 & 0 & 0 & -1 & 1 & 0 & 0 \\ \hline 0 & 0 & 0 & 0 & 1 & 0 & 0 & 1 & -1 & 0 & 0 & 0 & 0 \\ 0 & 0 & 0 & 1 & 0 & -1 & 0 & 0 & 1 & 0 & 0 & 0 & 0 \\ 0 & 0 & 0 & 0 & 1 & 1 & -1 & 0 & 0 & 0 & 0 & 0 & 0 \\ 0 & 0 & 0 & 1 & 0 & 0 & 1 & -1 & 0 & 0 & 0 & 0 & 0\end{array}\right)$.

The eigenvalues are: $-\sqrt{6},-\sqrt{2}, 0, \sqrt{2}, \sqrt{6}$ with multiplicities $3,3,1,3,3$, respectively.

In matrix form, the Laplacian is given by the square of the Dirac matrix:

$\frac{1}{2}\left(\begin{array}{ccc|cccccc|ccccc}4 & -2 & -2 & 0 & 0 & 0 & 0 & 0 & 0 & 0 & 0 & 0 & 0 \\ -2 & 4 & -2 & 0 & 0 & 0 & 0 & 0 & 0 & 0 & 0 & 0 & 0 \\ -2 & -2 & 4 & 0 & 0 & 0 & 0 & 0 & 0 & 0 & 0 & 0 & 0 \\ \hline 0 & 0 & 0 & 4 & -2 & 0 & 0 & 0 & 0 & 0 & 0 & 0 & 0 \\ 0 & 0 & 0 & -2 & 4 & 0 & 0 & 0 & 0 & 0 & 0 & 0 & 0 \\ 0 & 0 & 0 & 0 & 0 & 4 & 0 & 2 & 0 & 0 & 0 & 0 & 0 \\ 0 & 0 & 0 & 0 & 0 & 0 & 4 & 0 & 2 & 0 & 0 & 0 & 0 \\ 0 & 0 & 0 & 0 & 0 & 2 & 0 & 4 & 0 & 0 & 0 & 0 & 0 \\ 0 & 0 & 0 & 0 & 0 & 0 & 2 & 0 & 4 & 0 & 0 & 0 & 0 \\ \hline 0 & 0 & 0 & 0 & 0 & 0 & 0 & 0 & 0 & 3 & -1 & 1 & -1 \\ 0 & 0 & 0 & 0 & 0 & 0 & 0 & 0 & 0 & -1 & 3 & -1 & 1 \\ 0 & 0 & 0 & 0 & 0 & 0 & 0 & 0 & 0 & 1 & -1 & 3 & -1 \\ 0 & 0 & 0 & 0 & 0 & 0 & 0 & 0 & 0 & -1 & 1 & -1 & 3\end{array}\right)$.

A third script for $\mathbb{R P}^{2}$ is in the spirit of the torus and the Klein bottle, as given in Figure 7. We obtain:

$$
\begin{array}{ll}
\partial l_{1}=p_{1}-p_{0}, & \partial l_{2}=p_{4}-p_{1}, \\
\partial l_{3}=p_{2}-p_{4}, & \partial l_{4}=p_{0}-p_{2}, \\
\partial l_{5}=p_{3}-p_{2}, & \partial l_{6}=p_{2}-p_{3}, \\
\partial l_{7}=p_{1}-p_{3}, & \partial l_{4}=p_{3}-p_{1},
\end{array}
$$




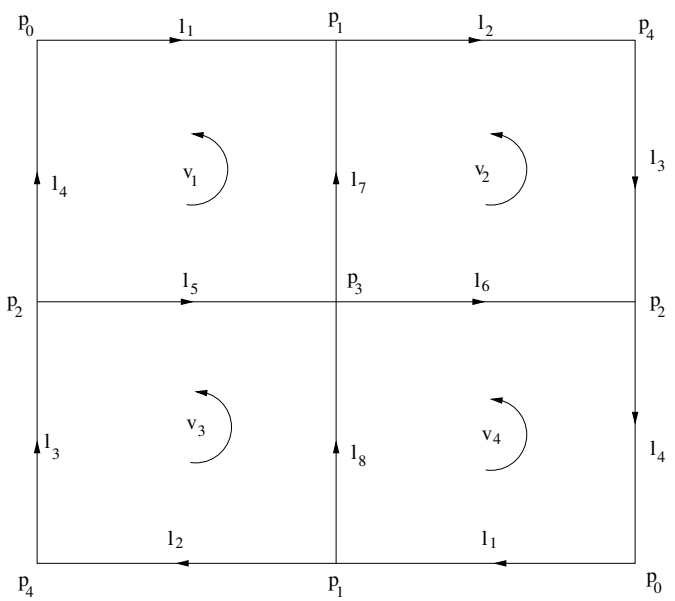

FiguRE 7. A third projective script

and

$$
\begin{aligned}
& \partial v_{1}=l_{5}+l_{7}-l_{1}-l_{4}, \\
& \partial v_{2}=l_{6}-l_{3}-l_{2}-l_{7}, \\
& \partial v_{3}=-l_{2}+l_{8}-l_{5}-l_{3}, \\
& \partial v_{4}=-l_{1}-l_{4}-l_{6}-l_{8} .
\end{aligned}
$$

We obtain

$$
\sum_{j=1}^{4} \partial v_{j}=-2\left(l_{1}+l_{2}+l_{3}+l_{4}\right) .
$$

Denoting the line sum $l_{1234}:=\left(l_{1}+l_{2}+l_{3}+l_{4}\right)$ and $\gamma$ the sum of all $v_{j}$, then $l_{1234}$ is a representing the non-zero element of $\mathcal{H}_{1}\left(\mathfrak{S}_{\mathbb{R P}^{2}}\right)$, and $\gamma$ is not a cycle, as $\partial \gamma=-2 l_{1234}$, i.e. we get again:

\begin{tabular}{|c|c|c|c|c|c|c|c|c|c|c|c|c|c|c|c|c|}
\hline 0 & 0 & 0 & 0 & 0 & -1 & 0 & 0 & 1 & 0 & 0 & 0 & 0 & 0 & 0 & 0 & 0 \\
\hline 0 & 0 & 0 & 0 & 0 & 1 & -1 & 0 & 0 & 0 & 0 & 1 & -1 & 0 & 0 & 0 & 0 \\
\hline 0 & 0 & 0 & 0 & 0 & 0 & 0 & 1 & -1 & -1 & 1 & 0 & 0 & 0 & 0 & 0 & 0 \\
\hline 0 & 0 & 0 & 0 & 0 & 0 & 0 & 0 & 0 & 1 & -1 & -1 & 1 & 0 & 0 & 0 & 0 \\
\hline 0 & 0 & 0 & 0 & 0 & 0 & 1 & -1 & 0 & 0 & 0 & 0 & 0 & 0 & 0 & 0 & 0 \\
\hline-1 & 1 & 0 & 0 & 0 & 0 & 0 & 0 & 0 & 0 & 0 & 0 & 0 & -1 & 0 & 0 & -1 \\
\hline 0 & -1 & 0 & 0 & 1 & 0 & 0 & 0 & 0 & 0 & 0 & 0 & 0 & 0 & -1 & -1 & 0 \\
\hline 0 & 0 & 1 & 0 & -1 & 0 & 0 & 0 & 0 & 0 & 0 & 0 & 0 & 0 & -1 & -1 & 0 \\
\hline 1 & 0 & -1 & 0 & 0 & 0 & 0 & 0 & 0 & 0 & 0 & 0 & 0 & -1 & 0 & 0 & -1 \\
\hline 0 & 0 & -1 & 1 & 0 & 0 & 0 & 0 & 0 & 0 & 0 & 0 & 0 & 1 & 0 & -1 & 0 \\
\hline 0 & 0 & 1 & -1 & 0 & 0 & 0 & 0 & 0 & 0 & 0 & 0 & 0 & 0 & 1 & 0 & -1 \\
\hline 0 & 1 & 0 & -1 & 0 & 0 & 0 & 0 & 0 & 0 & 0 & 0 & 0 & 1 & -1 & 0 & 0 \\
\hline 0 & -1 & 0 & 1 & 0 & 0 & 0 & 0 & 0 & 0 & 0 & 0 & 0 & 0 & 0 & 1 & -1 \\
\hline 0 & 0 & 0 & 0 & 0 & -1 & 0 & 0 & -1 & 1 & 0 & 1 & 0 & 0 & 0 & 0 & 0 \\
\hline 0 & 0 & 0 & 0 & 0 & 0 & -1 & -1 & 0 & 0 & 1 & -1 & 0 & 0 & 0 & 0 & 0 \\
\hline 0 & 0 & 0 & 0 & 0 & 0 & -1 & -1 & 0 & -1 & 0 & 0 & 1 & 0 & 0 & 0 & 0 \\
\hline 0 & 0 & 0 & 0 & 0 & -1 & 0 & 0 & -1 & 0 & -1 & 0 & -1 & 0 & 0 & 0 & 0 \\
\hline
\end{tabular}

$$
\mathcal{H}_{1}\left(\mathfrak{S}_{\mathbb{R} \mathbb{P}^{2}}\right) \simeq \mathbb{Z}_{2}, \quad \mathcal{H}_{2}\left(\mathfrak{S}_{\mathbb{R}^{2}}\right)=0
$$

The cohomology is calculated in a similar way.

Similar to the computations above for the Klein bottle, we obtain the following matrix form for the discrete Dirac operator on $\mathbb{R P}^{2}$ : 
with eigenvalues $-2 \sqrt{2},-\sqrt{5+\sqrt{5}},-\sqrt{5-\sqrt{5}},-2,-\sqrt{2}, 0, \sqrt{2}, 2, \sqrt{5-\sqrt{5}}$, $\sqrt{5+\sqrt{5}}, 2 \sqrt{2}$ with multiplicities $1,2,2,3,1,3,2,2,1$, respectively.

The Laplacian for this script depicting $\mathbb{R P}^{2}$ is given by:

\begin{tabular}{|c|c|c|c|c|c|c|c|c|c|c|c|c|c|c|c|c|}
\hline 2 & -1 & -1 & 0 & 0 & 0 & 0 & 0 & 0 & 0 & 0 & 0 & 0 & 0 & 0 & 0 & 0 \\
\hline-1 & 4 & 0 & -2 & 1 & 0 & 0 & 0 & 0 & 0 & 0 & 0 & 0 & 0 & 0 & 0 & 0 \\
\hline-1 & 0 & 4 & -2 & -1 & 0 & 0 & 0 & 0 & 0 & 0 & 0 & 0 & 0 & 0 & 0 & 0 \\
\hline 0 & -2 & -2 & 4 & 0 & 0 & 0 & 0 & 0 & 0 & 0 & 0 & 0 & 0 & 0 & 0 & 0 \\
\hline 0 & -1 & -1 & 0 & 2 & 0 & 0 & 0 & 0 & 0 & 0 & 0 & 0 & 0 & 0 & 0 & 0 \\
\hline 0 & 0 & 0 & 0 & 0 & 4 & -1 & 0 & 1 & -1 & 1 & 0 & 0 & 0 & 0 & 0 & 0 \\
\hline 0 & 0 & 0 & 0 & 0 & -1 & 4 & 1 & 0 & 1 & -1 & 0 & 0 & 0 & 0 & 0 & 0 \\
\hline 0 & 0 & 0 & 0 & 0 & 0 & 1 & 4 & -1 & 0 & 0 & 1 & -1 & 0 & 0 & 0 & 0 \\
\hline 0 & 0 & 0 & 0 & 0 & 1 & 0 & -1 & 4 & 0 & 0 & -1 & 1 & 0 & 0 & 0 & 0 \\
\hline 0 & 0 & 0 & 0 & 0 & -1 & 1 & 0 & 0 & 4 & -2 & 0 & 0 & 0 & 0 & 0 & 0 \\
\hline 0 & 0 & 0 & 0 & 0 & 1 & -1 & 0 & 0 & -2 & 4 & 0 & 0 & 0 & 0 & 0 & 0 \\
\hline 0 & 0 & 0 & 0 & 0 & 0 & 0 & 1 & -1 & 0 & 0 & 4 & -2 & 0 & 0 & 0 & 0 \\
\hline 0 & 0 & 0 & 0 & 0 & 0 & 0 & -1 & 1 & 0 & 0 & -2 & 4 & 0 & 0 & 0 & 0 \\
\hline 0 & 0 & 0 & 0 & 0 & 0 & 0 & 0 & 0 & 0 & 0 & 0 & 0 & 4 & -1 & -1 & 2 \\
\hline 0 & 0 & 0 & 0 & 0 & 0 & 0 & 0 & 0 & 0 & 0 & 0 & 0 & -1 & 4 & 2 & -1 \\
\hline 0 & 0 & 0 & 0 & 0 & 0 & 0 & 0 & 0 & 0 & 0 & 0 & 0 & -1 & 2 & 4 & -1 \\
\hline 0 & 0 & 0 & 0 & 0 & 0 & 0 & 0 & 0 & 0 & 0 & 0 & 0 & 2 & -1 & -1 & 4 \\
\hline
\end{tabular}

Finally, we can obtain an $\mathbb{R P}^{2}$ by adding a rectangle $v_{3}$ to a Möbius strip $M$ (see subsection 5.1) with boundary

$$
\partial v_{3}=l_{2}+l_{3}+l_{4}+l_{5},
$$

as in Figure 8. It follows that

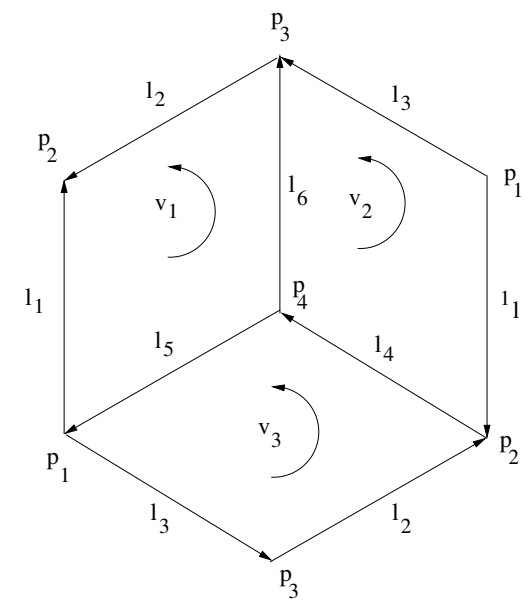

FiguRE 8. Projective plane obtained from a Möbius strip

$$
\partial\left(v_{1}+v_{2}+v_{3}\right)=2\left(l_{2}+l_{3}-l_{1}\right),
$$

which is the generator of $\mathcal{H}_{1}\left(\mathfrak{S}_{\mathbb{R}^{2}}\right)=\mathbb{Z}_{2}$.

The differential operator $d$ acts on this script as follows:

$$
\begin{aligned}
& d p_{1}=-l_{1}-l_{3}+l_{5}, \\
& d p_{2}=l_{1}+l_{2}-l_{4}, \\
& d p_{3}=-l_{2}+l_{3}+l_{6}, \\
& d p_{4}=l_{4}-l_{5}-l_{6},
\end{aligned}
$$


and

$$
\begin{gathered}
d l_{1}=-v_{1}-v_{2}, \quad d l_{2}=v_{1}+v_{3}, \quad d l_{3}=v_{2}+v_{3}, \quad d l_{4}=-v_{2}+v_{3}, \\
d l_{5}=-v_{1}+v_{3}, \quad d l_{6}=v_{1}-v_{2} .
\end{gathered}
$$

The resulting Dirac operator matrix for this script is:

$\left(\begin{array}{cccc|cccccc|ccc}0 & 0 & 0 & 0 & -1 & 0 & -1 & 0 & 1 & 0 & 0 & 0 & 0 \\ 0 & 0 & 0 & 0 & 1 & 1 & 0 & -1 & 0 & 0 & 0 & 0 & 0 \\ 0 & 0 & 0 & 0 & 0 & -1 & 1 & 0 & 0 & 1 & 0 & 0 & 0 \\ 0 & 0 & 0 & 0 & 0 & 0 & 0 & 1 & -1 & -1 & 0 & 0 & 0 \\ \hline-1 & 1 & 0 & 0 & 0 & 0 & 0 & 0 & 0 & 0 & -1 & -1 & 0 \\ 0 & 1 & -1 & 0 & 0 & 0 & 0 & 0 & 0 & 0 & 1 & 0 & 1 \\ -1 & 0 & 1 & 0 & 0 & 0 & 0 & 0 & 0 & 0 & 0 & 1 & 1 \\ 0 & -1 & 0 & 1 & 0 & 0 & 0 & 0 & 0 & 0 & 0 & -1 & 1 \\ 1 & 0 & 0 & -1 & 0 & 0 & 0 & 0 & 0 & 0 & -1 & 0 & 1 \\ 0 & 0 & 1 & -1 & 0 & 0 & 0 & 0 & 0 & 0 & 1 & -1 & 0 \\ \hline 0 & 0 & 0 & 0 & -1 & 1 & 0 & 0 & -1 & 1 & 0 & 0 & 0 \\ 0 & 0 & 0 & 0 & -1 & 0 & 1 & -1 & 0 & -1 & 0 & 0 & 0 \\ 0 & 0 & 0 & 0 & 0 & 1 & 1 & 1 & 1 & 0 & 0 & 0 & 0\end{array}\right)$

with eigenvalues $-2,0,2$ of multiplicites $6,1,6$, respectively. The Laplacian of this script is given by:

$\frac{1}{2}\left(\begin{array}{cccc|cccccc|ccc}3 & -1 & -1 & -1 & 0 & 0 & 0 & 0 & 0 & 0 & 0 & 0 & 0 \\ -1 & 3 & -1 & -1 & 0 & 0 & 0 & 0 & 0 & 0 & 0 & 0 & 0 \\ -1 & -1 & 3 & -1 & 0 & 0 & 0 & 0 & 0 & 0 & 0 & 0 & 0 \\ -1 & -1 & -1 & 3 & 0 & 0 & 0 & 0 & 0 & 0 & 0 & 0 & 0 \\ \hline 0 & 0 & 0 & 0 & 4 & 0 & 0 & 0 & 0 & 0 & 0 & 0 & 0 \\ 0 & 0 & 0 & 0 & 0 & 4 & 0 & 0 & 0 & 0 & 0 & 0 & 0 \\ 0 & 0 & 0 & 0 & 0 & 0 & 4 & 0 & 0 & 0 & 0 & 0 & 0 \\ 0 & 0 & 0 & 0 & 0 & 0 & 0 & 4 & 0 & 0 & 0 & 0 & 0 \\ 0 & 0 & 0 & 0 & 0 & 0 & 0 & 0 & 4 & 0 & 0 & 0 & 0 \\ 0 & 0 & 0 & 0 & 0 & 0 & 0 & 0 & 0 & 4 & 0 & 0 & 0 \\ \hline 0 & 0 & 0 & 0 & 0 & 0 & 0 & 0 & 0 & 0 & 4 & 0 & 0 \\ 0 & 0 & 0 & 0 & 0 & 0 & 0 & 0 & 0 & 0 & 0 & 4 & 0 \\ 0 & 0 & 0 & 0 & 0 & 0 & 0 & 0 & 0 & 0 & 0 & 0 & 4\end{array}\right)$.

\subsection{Connected sum of two projective planes}

First, we construct the following tight script $\mathfrak{S}_{2 \mathbb{R} \mathbb{P}^{2}}$ for a connected sum of two $\mathbb{R P}^{2}$, as in Figure 9. Topologically it is obtained from two circles, then one cuts a third "small" circle from each initial ones and glue them along their boundary (with the same orientation) to form the connected sum. In Figure 9 the two initial circles are: first $\left(l_{1}+l_{2}\right)$ from upper left corner identified with $l_{1}+l_{2}$ from bottom left corner - that gives a circle around the point $p_{1}$; the second circle is $l_{4}+l_{3}$ from the upper right corner identified with $l_{4}+l_{3}$ from lower right corner - a second circle around $p_{1}$. Finally, the gluing circle is $\left(l_{6}+l_{5}\right)$, also around $p_{1}$. It is well known that topologically $\mathbb{R} \mathbb{P}^{2} \sharp \mathbb{R P}^{2}$ is equivalent to a Klein bottle. We obtain the following script geometry:

$$
\begin{array}{ll}
\partial l_{1}=p_{2}-p_{1}, & \partial l_{2}=p_{1}-p_{2}, \\
\partial l_{3}=p_{1}-p_{3}, & \partial l_{4}=p_{3}-p_{1}, \\
\partial l_{5}=p_{1}-p_{0}, & \partial l_{6}=p_{0}-p_{1}, \\
\partial l_{7}=p_{0}-p_{1}, & \partial l_{8}=p_{1}-p_{0},
\end{array}
$$




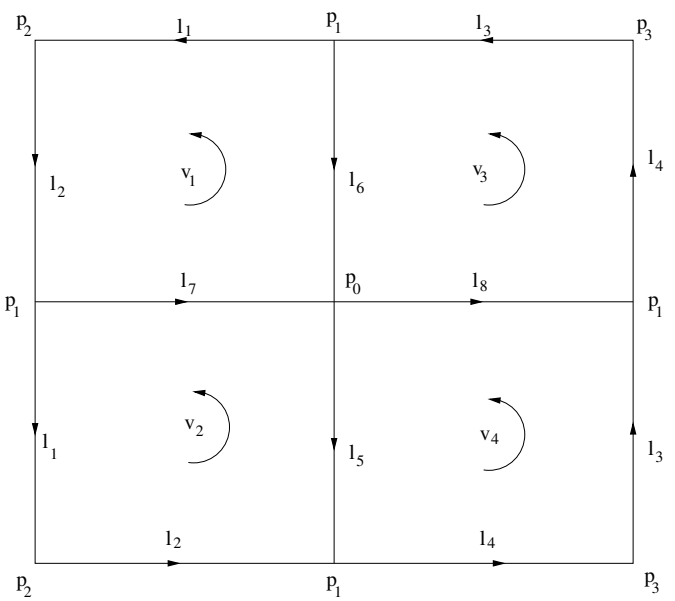

Figure 9. Connected sum of two projective planes

and

$$
\begin{aligned}
& \partial v_{1}=l_{1}+l_{2}-l_{6}+l_{7}, \\
& \partial v_{2}=l_{1}+l_{2}-l_{5}-l_{7}, \\
& \partial v_{3}=l_{3}+l_{4}+l_{6}+l_{8}, \\
& \partial v_{4}=l_{3}+l_{4}+l_{5}-l_{8} .
\end{aligned}
$$

Therefore,

$$
\sum_{j=1}^{2} \partial v_{j}=2\left(l_{1}+l_{2}+l_{3}+l_{4}\right),
$$

which lead to $\mathcal{H}_{1}\left(\mathfrak{S}_{2 \mathbb{R}^{2}}\right) \simeq \mathbb{Z} \oplus \mathbb{Z}_{2}$ and $\mathcal{H}_{2}\left(\mathfrak{S}_{2 \mathbb{R} \mathbb{P}^{2}}\right)=0$, same script holomology of a Klein bottle.

At the differential operator level, we obtain:

$$
\begin{aligned}
& d p_{0}=-l_{5}+l_{6}+l_{7}-l_{8}, \\
& d p_{1}=-l_{1}+l_{2}+l_{3}-l_{4}+l_{5}-l_{6}-l_{7}+l_{8}, \\
& d p_{2}=l_{1}-l_{2}, \quad d p_{3}=l_{4}-l_{3},
\end{aligned}
$$

and

$$
\begin{aligned}
d l_{1}=v_{1}+v_{2}, & d l_{2}=v_{1}+v_{2}, \\
d l_{3}=v_{3}+v_{4}, & d l_{4}=v_{3}+v_{4}, \\
d l_{5}=-v_{2}+v_{4}, & d l_{6}=-v_{1}+v_{3}, \\
d l_{7}=v_{1}-v_{2}, & d l_{8}=v_{3}-v_{4} .
\end{aligned}
$$


In matrix form, the Dirac operator for the $\mathbb{R P}^{2} \sharp \mathbb{R} \mathbb{P}^{2}$ script is:

$\left(\begin{array}{cccc|cccccccccccccc}0 & 0 & 0 & 0 & 0 & 0 & 0 & 0 & -1 & 1 & 1 & -1 & 0 & 0 & 0 & 0 \\ 0 & 0 & 0 & 0 & -1 & 1 & 1 & -1 & 1 & -1 & -1 & 1 & 0 & 0 & 0 & 0 \\ 0 & 0 & 0 & 0 & 1 & -1 & 0 & 0 & 0 & 0 & 0 & 0 & 0 & 0 & 0 & 0 \\ 0 & 0 & 0 & 0 & 0 & 0 & -1 & 1 & 0 & 0 & 0 & 0 & 0 & 0 & 0 & 0 \\ \hline 0 & -1 & 1 & 0 & 0 & 0 & 0 & 0 & 0 & 0 & 0 & 0 & 1 & 1 & 0 & 0 \\ 0 & 1 & -1 & 0 & 0 & 0 & 0 & 0 & 0 & 0 & 0 & 0 & 1 & 1 & 0 & 0 \\ 0 & 1 & 0 & -1 & 0 & 0 & 0 & 0 & 0 & 0 & 0 & 0 & 0 & 0 & 1 & 1 \\ 0 & -1 & 0 & 1 & 0 & 0 & 0 & 0 & 0 & 0 & 0 & 0 & 0 & 0 & 1 & 1 \\ -1 & 1 & 0 & 0 & 0 & 0 & 0 & 0 & 0 & 0 & 0 & 0 & 0 & -1 & 0 & 1 \\ 1 & -1 & 0 & 0 & 0 & 0 & 0 & 0 & 0 & 0 & 0 & 0 & -1 & 0 & 1 & 0 \\ 1 & -1 & 0 & 0 & 0 & 0 & 0 & 0 & 0 & 0 & 0 & 0 & 1 & -1 & 0 & 0 \\ -1 & 1 & 0 & 0 & 0 & 0 & 0 & 0 & 0 & 0 & 0 & 0 & 0 & 0 & 1 & -1 \\ \hline 0 & 0 & 0 & 0 & 1 & 1 & 0 & 0 & 0 & -1 & 1 & 0 & 0 & 0 & 0 & 0 \\ 0 & 0 & 0 & 0 & 1 & 1 & 0 & 0 & -1 & 0 & -1 & 0 & 0 & 0 & 0 & 0 \\ 0 & 0 & 0 & 0 & 0 & 0 & 1 & 1 & 0 & 1 & 0 & 1 & 0 & 0 & 0 & 0 \\ 0 & 0 & 0 & 0 & 0 & 0 & 1 & 1 & 1 & 0 & 0 & -1 & 0 & 0 & 0 & 0\end{array}\right)$

with eigenvalues

$-\sqrt{7+\sqrt{17}},-\sqrt{6},-\sqrt{7-\sqrt{17}},-2,-\sqrt{2}, 0, \sqrt{2}, 2, \sqrt{7-\sqrt{17}}, \sqrt{6}, \sqrt{7+\sqrt{17}}$

of multiplicities $1,1,1,2,2,2,2,2,1,1,1$, respectively.

The Laplace operator is given by:

$$
\begin{aligned}
& 2 \Delta_{2 \mathbb{R}^{2}} f=\left[\begin{array}{cccc}
4 & -4 & 0 & 0 \\
-4 & 8 & -2 & -2 \\
0 & -2 & 2 & 0 \\
0 & -2 & 0 & 2
\end{array}\right]\left[f_{0 j}\right]^{t}\left[p_{j}\right] \\
& +\left[\begin{array}{cccccccc}
4 & 0 & -1 & 1 & -2 & 0 & 1 & -1 \\
0 & 4 & 1 & -1 & 0 & -2 & -1 & 1 \\
-1 & 1 & 4 & 0 & 2 & 0 & -1 & 1 \\
1 & -1 & 0 & 4 & 0 & 2 & 1 & -1 \\
-2 & 0 & 2 & 0 & 4 & -2 & -1 & 1 \\
0 & 2 & 0 & 2 & -2 & 4 & 1 & -1 \\
1 & -1 & -1 & 1 & -1 & 1 & 4 & -2 \\
-1 & 1 & 1 & -1 & 1 & -1 & -2 & 4
\end{array}\right]\left[f_{1 j}\right]^{t}\left[l_{j}\right] \\
& +\frac{1}{2}\left[\begin{array}{cccc}
4 & 1 & -1 & 0 \\
1 & 4 & 0 & -1 \\
-1 & 0 & 4 & 1 \\
0 & -1 & 1 & 4
\end{array}\right]\left[f_{2 j}\right]^{t}\left[v_{j}\right]
\end{aligned}
$$

A different method of obtaining the connected sum $\mathbb{R} \mathbb{P}^{2} \sharp \mathbb{R} \mathbb{P}^{2}$ is done by attaching two Möbius bands on the same boundary, as the projective plane $\mathbb{R P}^{2}$ minus a disk is topological equivalent to a Möbius strip. Looking back at Figure 2, we attach to it a second Möbius band as in Figure 10. The two strips glued together on the same four points $p_{1}, p_{2}, p_{3}, p_{4}$, containing two more lines $l_{7}, l_{8}$ and two more planes $v_{3}, v_{4}$ as in Figure 10 . To the computations of subsection 5.1 we add the following boundaries:

$$
\begin{aligned}
\partial l_{7} & =p_{1}-p_{2}, \quad \partial l_{8}=p_{4}-p_{3}, \\
\partial v_{3} & =-l_{2}+l_{5}-l_{7}+l_{8}, \quad \partial v_{4}=-l_{3}+l_{4}-l_{7}-l_{8} .
\end{aligned}
$$




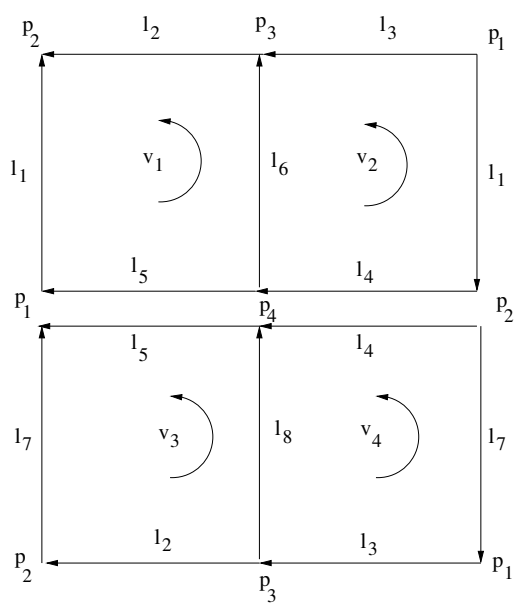

Figure 10. Connecting two Möbius strips

Here we note that

$$
\partial\left(v_{1}+v_{2}-v_{3}-v_{4}\right)=2\left(l_{7}-l_{1}\right),
$$

is the generator of the $\mathcal{H}_{1}\left(\mathfrak{S}_{\mathbb{R}^{2}}\right)=\mathbb{Z}_{2}$ homology. Note that we obtain the same script (different labelling) as the Klein bottle (see Figure 4), for which we performed all the necessary computations in subsection 5.3.

\section{Outlook}

In $[13,15]$ the authors present an approach to discrete differential modeling, which includes notions of discrete differential forms on simplexes and discrete manifolds, discrete boundary and co-boundary operators, discrete Hodge decomposition, and a discrete version of the Poincaré lemma. The same can and will be studied in the case of Script Geometry although some of necessary tools need to be developed since Script Geometry is a more loose concept than working with simplicial complexes.

In [14] the authors describe their approach to the theory of discrete exterior calculus (DEC). They introduce notions of discrete vector fields and operators acting on them, e.g. discrete divergence and curl, which has applications such as a discrete Hodge decomposition of 3D vector fields on irregular grids. A closely related work is discrete mechanics, where the main idea is to discretize the variational priciple itself rather than the Euler-Lagrange equations. The discretization is not intended on time only, DEC methods are used in spacially extended mechanics, i.e. classical field theory. Furthermore, this theory is also widely applied in discrete electromagnetism which is another field were we see applications of Script Geometry in the future. This is also closely linked with a principal question in finite element exterior calculus. 
Up to now the commutativity of the exterior derivative with bounded projections to sub-meshes was only being shown for simplicial decompositions of domains. From our point of view this is still a major drawback for applying this calculus to more general type of meshes. Here, Script Geometry could be the basis for a more general approach.

In $[10,11,12]$ a complete function theory has been established for a Dirac type operator on the grid $\mathbb{Z}^{n}$, including Taylor series, Fueter polynomials, and a discrete Cauchy-Kovalevskaya theorem. We look forward to relate this work to script geometry.

\section{Aknowledgement}

The first and second authors were supported by Portuguese funds through the CIDMA - Center for Research and Development in Mathematics and Applications, and the Portuguese Foundation for Science and Technology ("FCT-Fundação para a Ciência e a Tecnologia"), within project UID/MAT/ $0416 / 2013$.

\section{References}

\section{References}

[1] D. N. Arnold, R. S. Falk, R. Winther, "Finite element exterior Calculus, homological techniques, and applications", Acta Numerica 2006, 1-155.

[2] F. Baaske, S. Bernstein, H. De Ridder, F. Sommen, "On solutions of a discretized heat equation in discrete Clifford analysis", J. Diff. Eq. Appl. 20 (2), pp. 271-295.

[3] M. Belkin, J. Sun, Y. Wang, "Discrete Laplace operator on meshed surfaces", SCG08, College Park, Maryland, 2008

[4] F. Brackx, D. De Schepper, F. Sommen, L. Van de Voorde, "Discrete Clifford Analysis: A Germ of Function Theory", in: I. Sabadini, M. Shapiro, F. Sommen (eds.), Hypercomplex Analysis, Birkhäuser, 37-53, 2009.

[5] P. Cerejeiras, N. Faustino, N. Vieira, "Numerical Clifford analysis for nonlinear Schrödinger problem", Numerical Methods for Partial Differential Equations, 24(4) (2008) 1181-1202, 2008.

[6] P. Cerejeiras, U. Kähler, M. Ku, "Discrete Hilbert boundary value problems on half lattices", Journal of Difference Equations and Applications, 21 (12) (2015), 1277-1304.

[7] P. Cerejeiras, U. Kähler, M. Ku, F. Sommen, "Discrete Hardy spaces", Journal of Fourier Analysis and Applications, 20 (2014), 715-750.

[8] H. De Schepper, F. Sommen, L. Van de Voorde, "A Basic Framework for Discrete Clifford Analysis", Exp. Math. 18:4, 2009.

[9] H. De Ridder, "Discrete Clifford analysis: function theory and integral transforms", PhD-thesis, Ghent, 2013.

[10] H. De Ridder, H. De Schepper, F. Sommen, "Fueter polynomials in discrete Clifford analysis", Math. Zeit. 272 (1-2) (2012), pp. 253-268. 
[11] H. De Ridder, H. De Schepper, F. Sommen. "Taylor series expansion in discrete Clifford analysis", Compl. Anal. Oper. Th. 8 (2), (2013), pp. 485-511.

[12] H. De Ridder, H. De Schepper, F. Sommen. "The Cauchy-Kovalevskaya Extension Theorem in Discrete Clifford Analysis", Comm. Pure Appl. Math. 10 (4) (2011), 1097-1109.

[13] M. Desbrun, E. Kanso, Y. Tong, "Discrete Differential Forms for Computational Modeling", in: Discrete Differential Geometry, Volume 38 of the series Oberwolfach Seminars, 287-324, 2008.

[14] M. Desbrun, A.N. Hirani, J.E. Marsden, "Discrete Exterior Calculus for Variational Problems in Computer Vision and Graphics", in 42nd IEEE Conference on Decision and Control Proceedings (CDC '03), 42 (2003), 4902-4907.

[15] M. Desbrun, M. Leok, J.E. Marsden, "Discrete Poincaré lemma", Appl. Num. Math. 53 (2005), 231-248.

[16] A. Dimakis, F. Müller-Hoissen, "Discrete Differential Calculus, Graphs, Topologies and Gauge Theory", J. Math. Phys. 35, 6703-6735, 1994.

[17] N. Faustino, Discrete Clifford Analysis, PhD Thesis, University of Aveiro, 2008.

[18] N. Faustino, U. Kähler, "Fischer decomposition for difference Dirac operators" Adv. Appl. Clifford Algebras 17-1 (2007).

[19] N. Faustino, U. Kähler, F.Sommen, "Discrete Dirac operators in Clifford Analysis", Adv. Appl. Clifford Algebras 17:3 (2007).

[20] N. Faustino, G. Ren, "(Discrete) Almansi Type Decompositions: An umbral calculus framework based on osp (1|2) symmetries", Math. Meth. Appl. Sci. 11 (2011), 305-318.

[21] E. Forgy, U. Schreiber, "Discrete differential geometry on causal graphs", arXiv:math-ph/0407005v1, 2004.

[22] K. Gürlebeck, A. Hommel, "On finite difference potentials and their fundamental solutions", Adv. Appl.Clifford Algebras 11:2 (2001).

[23] K. Gürlebeck, A. Hommel, "On finite difference potentials and their applications in a discrete function theory", Math. Methods Appl. Sci. 25:16-18 (2002)

[24] P. Herholz, "General discrete Laplace operators on polygonal meshes", Diplomarbeit, Humboldt Univ. Berlin, 2012

[25] R. P. Isaacs, "A finite difference function theory", Univ. Nac. Tucumán, Revista A, 2, 177-201, 1941.

[26] I. Kanamori, N. Kawamoto, "Dirac-Kaehler Fermion from Clifford Product with Noncommutative Differential Form on a Lattice", Int. J. Mod. Phys. A19 (2004), 695-736.

[27] P. Leopardi, A. Stern, The abstract Hodge-Dirac operator and its stable discretization, arXiv:1401.1576, 2014.

[28] L. Lovasz, "Discrete Analytic Functions: An Exposition", Surveys in differential geometry, Vol. IX, 1-44, 2004.

[29] S. Smirnov, "Discrete Complex Analysis and Probability", Proceedings of the ICM 2010, Hyderabad, 2010, 1-27.

[30] A. Stern, "Discrete Differential Forms for Numerical Relativity", PCGM 2008. 
[31] M. Wardetzky, S. Mathur, F. Kälberer, E. Grinspun, "Discrete Laplace operators: no free lunch", Eurographics Symposium on Geometry Processing, 2007.

[32] J. Vaz, "Clifford-like calculus over lattices", Adv. Appl. Cliff. Alg., 7(1) (1997), 37-70.

P. Cerejeiras

Department of Mathematics, Universidade de Aveiro, P-3810-159 Aveiro, Portugal e-mail: pceres@ua.pt

U. Kähler

Department of Mathematics, Universidade de Aveiro, P-3810-159 Aveiro, Portugal e-mail: ukaehler@ua.pt

F. Sommen

Clifford Research Group, Department of Mathematical Analysis, Ghent University, Galglaan 2, B-9000 Ghent, Belgium

e-mail: fs@cage.ugent.be
A. Vajiac
Chapman University, One University Drive, Orange CA 92866
e-mail: avajiac@chapman.edu 